\title{
A Mechanism for Ionization of Nonvolatile Compounds in Mass Spectrometry: Considerations from MALDI and Inlet lonization
}

\author{
Sarah Trimpin, ${ }^{1}$ Beixi Wang, ${ }^{1}$ Ellen D. Inutan, ${ }^{1}$ Jing Li, ${ }^{1}$ Christopher B. Lietz, ${ }^{1}$ \\ Andrew Harron, ${ }^{2}$ Vincent S. Pagnotti, ${ }^{2}$ Diana Sardelis, ${ }^{2}$ Charles N. McEwen ${ }^{2}$ \\ ${ }^{1}$ Department of Chemistry, Wayne State University, Detroit, MI 48202, USA \\ ${ }^{2}$ Department of Chemistry and Biochemistry, University of the Sciences, Philadelphia, PA 19104, USA
}

\begin{abstract}
Mechanistic arguments relative to matrix-assisted laser desorption/ionization (MALDI) mass spectrometry (MS) address observations that predominately singly charged ions are detected. However, recently a matrix assisted laser ablation method, laserspray ionization (LSI), was introduced that can use the same sample preparation and laser as MALDI, but produce highly charged ions from proteins. In MALDI, ions are generated from neutral molecules by the photon energy provided to a matrix, while in LSI ions are produced inside a heated inlet tube linking atmospheric pressure and the first vacuum region of the mass spectrometer. Some LSI matrices also produce highly charged ions with MALDI ion sources operated at intermediate pressure or high vacuum. The operational similarity of LSI to MALDI, and the large difference in charge states observed by these methods, provides information of fundamental importance to proposed ionization mechanisms for LSI and MALDI. Here, we present data suggesting that the prompt and delayed ionization reported for vacuum MALDI are both fast processes relative to producing highly charged ions by LSI. The energy supplied to produce these charged clusters/droplets as well as their size and time available for desolvation are determining factors in the charge states of the ions observed. Further, charged droplets/clusters may be a common link for ionization of nonvolatile compounds by a variety of MS ionization methods, including MALDI and LSI.
\end{abstract}

Key words: Ionization mechanism, Laserspray ionization, LSI, MALDI, ESI, MAII, SAll, Multiply charged ions, Singly charged ions, Clusters, Charged droplets, Superheating, Matrix, Decomposition, Mechanism, Ionization, Charged clusters, Chemical ionization model, Gas phase model, Matrix assisted inlet ionization, Solvent assisted inlet ionization, Photochemical ionization

\section{Introduction}

$\mathrm{N}$ umerous mechanisms have been proposed for matrixassisted laser desorption/ionization (MALDI) mass

Electronic supplementary material The online version of this article (doi:10.1007/s13361-012-0414-y) contains supplementary material, which is available to authorized users.

Correspondence to: Sarah Trimpin; e-mail: strimpin@chem.wayne.edu, Charles McEwen; e-mail: c.mcewen@usp.edu spectrometry (MS) over the past 25 years [1-11]. The two most commonly cited are the two-step gas-phase photochemical ionization $[5,11]$ and the cluster models $[3,9,10]$. A recent publication by Jaskolla and Karas [2] discussed proof that both the photochemical and the most recent 'lucky survivor' (cluster) models are correct, at least for relatively small molecules. Any mechanism must take into account that similar MALDI mass spectra with a preponderance of singly charged ions are obtained using ultraviolet (UV) and infrared (IR) laser ablation, and at least for relatively low 
molecular weight compounds similar mass spectra are observed at low pressure (LP), intermediate pressure (IP), and atmospheric pressure (AP) [11-16]. The similar results, including the ability to desorb/ionize nonvolatile compounds, likely involve common mechanistic components. Here, we define MALDI as the ionization events and instrumental conditions from laser ablation of a matrix/analyte sample that lead to the observation of a preponderance of singly charged ions.

Recently, laser ablation at AP using a laser and common MALDI matrices prepared identical to methods used in MALDI resulted in formation of highly charged ions similar to electrospray ionization (ESI) $[17,18]$ even for proteins as large as bovine serum albumin (BSA), for which up to 67 charges were detected [19]. These results are significantly different from those reported for AP or vacuum MALDI [20]. The new ionization method called laserspray ionization (LSI) (Scheme S1A) has elements similar to MALDI and ESI, but is neither $[17,18]$. The commonality of MALDI $[21,22]$ and LSI $[17,18]$ is that ionization is matrix assisted, frequently using the same matrix compounds, sample preparation, and laser, and capable of producing gas-phase ions from nonvolatile compounds such as proteins directly from the solid state. Contrary to MALDI, LSI does not require absorption at the laser wavelength by the matrix as identical mass spectra are produced using physical means of transferring the matrix/analyte into the heated mass spectrometer inlet in a method termed matrix assisted inlet ionization (MAII) (Scheme S1B) [23]. Solvent assisted inlet ionization (SAII) (Scheme S1C) [24] is the liquid introduction variant of MAII capable of being coupled to liquid chromatography [25] even at nanoliter flow rates [26]. A brief discussion of the two-step photochemical ionization and cluster models for MALDI, and a background discussion of inlet ionization are presented below.

\section{The Two-Step Photochemical Ionization Model for MALDI}

In the two-step photochemical model, ionization is initiated by photoionization. Such a mechanism was suggested in early MALDI work [22, 27]. Knochenmuss [5] has refined the gas-phase mechanism, and he has been a main proponent of a chemical ionization process for MALDI in which matrix molecules ionized by photons from the laser pulse subsequently ionize analyte molecules in ion-molecule reactions. A difficulty of this mechanism is that the energy of a single photon, or for some matrix compounds even two photons, is insufficient for ionization of matrix molecules, and a two photon process with energy pooling has been proposed [5].

Insufficiencies of the gas-phase photochemical model have led to other proposed mechanisms [3, 4, 9, 10]. For example, IR-MALDI and UV-MALDI produce similar results, but the photon energy available for photoionization using IR lasers would seem to necessitate a lower probability three photon ionization process $[1,10]$, and even in UVMALDI two photon ionization has been considered problematic [6]. An additional issue is how the nonvolatile and high mass molecules become suspended in the gas phase for subsequent ionization. Vaporization is not expected, with for example proteins, so an alternative mechanism is required to 'lift' the nonvolatile analyte molecules into the gas phase. One suggested mechanism involving matrix assistance is rapid disintegration of the ablated volume into small particles that upon evaporation or sublimation of the matrix suspend the analyte molecules in the gas-phase [28]. On the other hand, the gas-phase model predicts the low charge state distribution of ions observed in MALDI. This is because the probability of gas-phase collision/ionization events between ions of like charge is low except where one of the colliding ions is sufficiently large that the distance between the charges minimizes the repulsive forces leading to the expectation of peptides being singly charged while large proteins may carry one to several charges, as is observed.

\section{The Cluster Mechanism in MALDI}

Prior to MALDI, Vestal [29] proposed a unified mechanism for ionization of nonvolatile compounds. This mechanism involved charging of droplets during the local disintegration of a surface. He envisioned a solid or liquid surface, which, upon ablation by particles or radiation, produces nano-droplets or clusters charged by a statistical process as previously described by Dodd [30]. The initial energy input is rapid, and for solids produce local melting with superheating of the fluid. A nucleation event produces the rapid energy release initiating charging of the resulting nano-droplets or clusters. Ions are released from the gas phase charged nano-droplets or clusters by the ion evaporation mechanism described by Iribarne and Thompson [31].

It was, therefore, anticipated that charged droplets may be involved in MALDI. Hereafter, we use the term droplets to mean all but small numbers of molten or solid matrix molecules, in which case we will use the term clusters. Hillenkamp, in discussions with John Fenn, suggested the possibility that MALDI and ESI might have similar mechanistic origins [32], but the first experimental evidence for clusters in MALDI were published by Kinsel et al. [33]. To explain results obtained studying the dynamics of ionization in MALDI, these authors proposed three possible scenarios, one involving emission of ions from clusters in a process analogous to ESI. Zhigilei and Garrison [34], using a breathing sphere model, predicted laser ablation of matrix droplets. Krutchinsky and Chait [35] proposed that the background ions observed in MALDI are, at least in part, due to matrix clusters. The most notable cluster mechanism, known as the 'lucky survivor' model, was proposed by Karas et al. [10]. Fournier et al. [9] reported delayed extraction experiments in which the relationship between 
the delay time and the ion flight time indicated that MALDI ions originate from clusters. These authors surmised from the experimental data the approximate size of the clusters that produce ions observed in MALDI time-of-flight (TOF) instruments and proposed that they are ablated without charge, but charging occurred on a nanosecond time scale. The formation of ablated clusters or droplets in the MALDI process is no longer an issue as they have been modeled [6, $34,36]$ and experimentally observed [37-40]. However, at issue is the importance of clusters in formation of the ions observed during MALDI analyses [2-4, 6, 8-10, 35, 41]. Knochenmuss has suggested that it is at best a minor contributor to ionization [6].

A problem with the cluster mechanism is how singly charged ions are produced. As noted above, it is well recognized that the cluster model is potentially related to ESI $[3,8,32,33]$ and, thus, multiple charging of clusters may be a possible outcome. However, Vestal's unified ionization model proposed in 1983, based on a charged droplet/cluster model, encompasses fast atom bombardment (FAB), secondary ion mass spectrometry (SIMS), laser desorption (LD), field desorption (FD), and thermospray ionization (TSI), all methods that produce abundant singly charged ions [29]. Karas and Kruger [8] suggested that singly charged ions observed in MALDI originate from clusters by separation of a single ion pair: separation of more than one ion pair was thought to be less favorable, both from a statistical and energetic standpoint. Alves et al. [3] and Fournier et al. $[9,41]$ using a MALDI-TOF mass spectrometer studied parameters affecting the formation of charge states greater than one in proteins. They suggested that two ionization mechanisms exist for formation of ions from highly charged clusters, a 'soft', or low energy, endothermic desolvation which involves loss of neutral matrix molecules and results in multiply charged analyte ions, and a 'hard' endothermic desolvation in which matrix ions are lost from the charged clusters creating singly charged analyte ions upon complete matrix evaporation.

\section{The Ion Formation Mechanism in LSI}

With any scientific discovery, the facts and knowledge of the underlying processes come piecemeal so that a complete understanding is not available with the early publications. LSI was initially thought to be a MALDI process [18] since the matrix and laser used were the same used in MALDI. Later it was discovered that the laser was unnecessary and ionization occurred in the heated inlet of the mass spectrometer [23]. Further experiments also demonstrate that inlet ionization is too narrow a definition because highly charged ions are produced from LSI matrix compounds under vacuum conditions using ion sources and lasers built for MALDI and without the availability of a heated inlet.

To simplify the discussion in this paper, we will use similar terms to distinguish LSI methods at different pressure as is used with MALDI. Thus, laserspray ionization inlet (LSII) used in other publications will be AP-LSI, laserspray ionization vacuum (LSIV) at IP will be IP-LSI, and at LP will be LSI-TOF to agree with AP-MALDI, IPMALDI, and MALDI-TOF. While AP-LSI is an inlet ionization method, IP-LSI and LSI-TOF are used here to represent the formation of highly charged ions similar to those produced in AP-LSI but using laser ablation of a matrix/analyte mixture under vacuum conditions just as in IP-MALDI and MALDI-TOF. LSI will represent the production of stable higher charge state ions not traditionally observed in MALDI.

Inlet ionization (Scheme S1) does not require externally applied voltage, photons, ions, or electrons to produce ESIlike mass spectra [23]. The commonly used UV MALDI matrix, 2,5-dihydroxyacetophenone (2,5-DHAP), produces nearly identical highly charged ions from laser ablation initiated at AP or IP (Scheme S1D) [42]. Because AP and IP results for LSI can have nearly identical charge state distributions using the same matrix (2,5-DHAP)/analyte mixture [42, 43], we assume that ionization in this case is governed by similar mechanisms. In AP-LSI, the function of the laser is to ablate the matrix/analyte mixture in a way that some of the ablated material enters the entrance aperture of the mass spectrometer. Ionization of the matrix/analyte particles/droplets, and subsequent formation of the bare analyte ions, occurs at sub-ambient conditions in the heated inlet tube [23]. By employing 2-nitrophloroglucinol (2NPG), one of several new LSI matrix compounds [44], multiple charging under IP conditions using the SYNAPT G2 MALDI source or LP conditions using MALDI-TOF mass spectrometers was recently demonstrated [45]. Charge states as high as +9 were observed for ubiquitin in a MALDI-TOF instrument and these high charge state ions are sufficiently stable to pass through the TOF reflectron. Multiply charged ions are observed with $\alpha$-cyano-4-hydroxycinnamic acid (CHCA), but with a somewhat lower charge state distribution than observed with 2-NPG and few of these ions pass through the reflectron indicating metastable decay occurs in the linear flight tube $[3,46]$.

A gas-phase ion-molecule mechanism for formation of highly charged ions in LSI can be ruled out as there is no experimental evidence in the literature suggesting that highly charged ions are produced by gas-phase ion-molecule reactions. Thus, the gas phase ions observed with LSI must originate directly from solids or liquids. Liquid droplets and solid particles of matrix are produced by laser ablation at AP $[39,47]$. Ion formation in LSI, similar to ESI, is thought to involve a two-step process. Highly charged matrix/analyte droplets are first produced and then evaporation of the matrix increases the charge density on the shrinking droplets leading to eventual ion formation by the ion evaporation [31] or charge residue [48] models similar to ESI. Note that matrix ions, as well as analyte ions, can be produced in this process. In LSI, volatile analyte can also be vaporized as gas phase neutral molecules and ionized in a chemical ionization 
process, presumably in collisions with the ionized matrix. As is the case with conventional sublimation techniques used for purification, evaporative processes are assisted by heat and vacuum. Here, we discuss mechanistic consideration relative to production of multiply and singly charged ions of nonvolatile compounds following laser ablation of a matrix/ analyte sample.

\section{Experimental}

\section{Materials}

2,5-Dihydroxybenzoic acid (2,5-DHB, $98 \%$ purity), CHCA, $98 \%$, sinapinic acid (SA, $99 \%$ purity), 2-NPG, $95 \%$, 4,6dinitrophloroglucinol (2,4-DNPG, $97 \%$ ), insulin (from bovine pancreas), ubiquitin (from bovine erythrocytes), lysozyme (from chicken egg white), myoglobin (from equine heart) and carbonic anhydrase (from bovine erythrocytes) were purchased from Sigma Aldrich Inc. (St. Louis, MO, USA). Myoglobin powder used in the direct inlet ionization experiment was made by grinding the protein as received using a mortar and pestle. Angiotensin I (Ang I) was purchased from American Peptide Co. (Sunnyvale, CA, USA) and $N$-acetylated myelin basic protein fragment (MBP) from Anaspec (Fremont, CA, USA). The O-GlcNAc peptide KKFELLP(gT)PPLSPSRR was provided by Professor Donald Hunt (University of Virginia) 2,5-DHAP, $97 \%$ purity, acetonitrile (ACN), and formic acid (FA) were obtained from Fisher Scientific Inc. (Pittsburgh, PA, USA). HPLC grade methanol $(\mathrm{MeOH})$ and water, as well as trifluoroacetic acid (TFA) were purchased from EMD Chemicals (Gibbstown, NJ, USA). Acetic acid was obtained from Mallinckrodt Chemicals (Phillipsburg, NJ, USA). Lavaquin was taken directly from a capsule of the prescription drug. Plain microscopy glass slides were purchased from Gold Seal Products (Portsmouth, NH, USA).

\section{Sample Preparation}

Stock solutions of Ang I, MBP, ubiquitin, lysozyme, myoglobin, and carbonic anhydrase were prepared in water and bovine insulin solution in $\mathrm{MeOH} /$ water with $1 \%$ acetic acid. Approximately $5 \mathrm{mg}$ of each matrix was used and individually prepared as saturated solutions in 50:50 ACN/ water for 2,5-DHAP $(150 \mu \mathrm{L}$, warmed) and 2-NPG $(100 \mu \mathrm{L})$, and in $50: 50 \mathrm{ACN} /$ water with $0.1 \%$ TFA for CHCA $(500 \mu \mathrm{L}), 2,5-\mathrm{DHB}(250 \mu \mathrm{L})$, and SA $(400 \mu \mathrm{L})$.

\section{AP-LSI and MAII Interfaced with LTQ and Orbitrap Exactive Mass Spectrometers}

A Thermo Fisher Scientific LTQ (San Jose, CA, USA) or an Orbitrap Exactive (Bremen, Germany) mass spectrometer was used to perform AP-LSI and MAII experiments. The ion max source was removed as described in previous studies [23]. Briefly, for AP-LSI, a nitrogen laser operated at
$337 \mathrm{~nm}$ wavelength (Spectra Physics VSL-337ND-S, Mountain View, CA, USA) was aligned $180^{\circ}$ to the mass spectrometer ion entrance and focused onto the sample in transmission geometry. The AP to vacuum ion transfer inlet capillary tube was heated from 50 to $450{ }^{\circ} \mathrm{C}$. For MAII, the same acquisition conditions were used as with AP-LSI but without the use of the laser and the analyte/matrix crystals were transferred into the ion transfer inlet capillary tube by methods described previously [23].

\section{AP-LSI Interfaced to a IMS-MS SYNAPT G2 Mass Spectrometer}

A nanoESI source was used on a Waters SYNAPT G2 mass spectrometer instrument to perform AP-LSI as previously described $[49,50]$. Scan time was set at 1 scan per s and up to $1 \mathrm{~min}$ acquisition time. MassLynx software was used to process the data and Driftscope ver. 2.2 was used to extract the 2-dimensional drift time vs. mass-to-charge $(\mathrm{m} / \mathrm{z})$ data.

\section{IP-LSI and IP-MALDI Interfaced to a SYNAPT G2 Mass Spectrometer}

A Waters SYNAPT G2 mass spectrometer (Manchester, UK) equipped with a commercial MALDI source and operated with a Nd:YAG laser $(355 \mathrm{~nm}, 200 \mathrm{~Hz})$ was used to carry-out IP-LSI as previously described [42]. Briefly, acquisitions were performed in positive ion sensitivity mode using the following LSI settings: $0 \mathrm{~V}$ on the sample plate holder, $10 \mathrm{~V}$ "extraction," $10 \mathrm{~V}$ "hexapole bias," and $5 \mathrm{~V}$ "aperture $0 . "$ The source pressure reading was at $0.214 \mathrm{mbar}$ and the drift cell pressure $3.29 \mathrm{mbar}$, identical to MALDI. The laser fluence used ranged from 4 to $21 \mathrm{~J} / \mathrm{cm}^{2}$ at $200 \mathrm{~Hz}$ firing rate. For the MALDI settings, the major difference is that sample plate voltage was set to $20 \mathrm{~V}$, Triwave trap DC bias to $150 \mathrm{~V}$, and higher laser fluence was used.

\section{LSI-TOF and MALDI-TOF on an UltrafleXtreme Mass Spectrometer}

A Bruker UltrafleXtreme mass spectrometer (Bremen, Germany) equipped with a Nd:YAG laser (355 nm, $1000 \mathrm{~Hz}$ ) with positive ion detection in reflectron and linear modes was used. The reflectron positive (RP) ion mode settings for ion source voltage (ISV) 1, ISV 2, lens voltage (LV), reflectron voltage (RV), and pulsed ion extraction (PIE) delay were $25.0,22.5,7.50,26.5 \mathrm{kV}$, and $90 \mathrm{~ns}$, respectively. Up to 1000 laser shots per mass spectrum were acquired at $1000 \mathrm{~Hz}$ frequency with 200 or 500 laser shot increments on the UltrafleXtreme. The laser attenuation ranged from $35 \%$ to $80 \%$ depending on the type of matrix used. 


\section{Results and Discussion}

\section{Production of Highly Charged Ions}

The charge state distribution observed in MALDI is compound and matrix dependent, but typically for any matrix the charge states, especially for larger molecules such as proteins and polymers, are low relative to ESI. On the other hand, using similar matrix preparation and laser ablation conditions as in MALDI, LSI [17, 18] produces abundant highly charged ions similar to ESI under AP [51] and IP [52] conditions. While the initial conditions in LSI and MALDI are similar, the results are drastically different. Figure 1a and $\mathrm{b}$ of the peptide MBP (MW 1833.2) obtained using the commercial IP-MALDI source on a SYNAPT G2 demonstrates the ability to produce either exclusively multiply charged or exclusively singly charged mass spectra using the same mass spectrometer and ion source, and the same matrix (2,5-DHAP)/analyte (MBP) sample spot for analysis. Figure 1a shows charge states +2 to +4 while Figure $1 \mathrm{~b}$ shows only charge state +1 of MBP. The principle instrumental differences are that Figure 1a is obtained under conditions tuned for multiply charged ions in ESI but operated at IP and uses lower laser fluence and lower ion acceleration voltage than used for obtaining singly charged ions (Figure 1b) in which the instrument is operated using 'factory' conditions for MALDI operation obtained by tuning using the matrix CHCA. Mass spectra observed using AP-LSI and the same 2,5-DHAP matrix and MBP analyte are nearly identical to Figure 1a. These results show that under vacuum MALDI conditions, multiply charged ions can be observed similar to those observed in ESI and AP-LSI provided proper instrumentation, tune conditions,

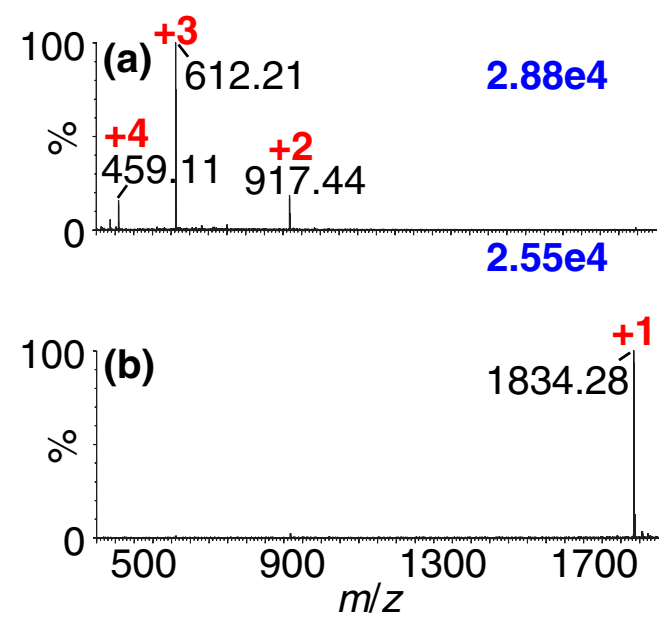

Figure 1. IP-LSI mass spectra of $N$-acetylated myelin basic protein fragment (MBP, MW 1833) with 2,5-DHAP matrix acquired using the Waters SYNAPT G2 mass spectrometer intermediate pressure MALDI ion source. (a) ESI tune, sample plate $0 \mathrm{~V}$, extractor lens $10 \mathrm{~V}$, hexapole bias $10 \mathrm{~V}$, and laser power of $5 \mathrm{~J} / \mathrm{cm}^{2}$, and (b) MALDI tune, sample plate $20 \mathrm{~V}$, hexapole bias $10 \mathrm{~V}$, extractor lens $10 \mathrm{~V}$, and a laser power of $15 \mathrm{~J} / \mathrm{cm}^{2}$ laser fluence and matrix are employed. For clarity, we refer to the conditions that produce multiply charged ions as IPLSI conditions, and those that produce singly charged ions as IP-MALDI conditions. These observations have implications for mechanistic aspects in MALDI.

A further interesting observation is that $[\mathrm{M}+\mathrm{H}]^{+}$ions are produced from low molecular weight compounds even without a matrix by inserting a neat particle of the material into the AP entrance of a heated inlet tube linking a high to a low pressure region (Scheme $\mathrm{S} 1$ ). This is shown for the drug lavaquin in Figure 2a, but more striking, pure myoglobin powder, placed into the heated inlet produces the multiply charged ESI-like mass spectrum shown in Figure 2b. Ions for both holo- and apo-myoglobin are observed suggesting this process is reasonably soft even though the inlet tube was heated to $425^{\circ} \mathrm{C}$ (measured at the heater block). No voltage, laser, or matrix is necessary to produce ions in the mass spectrometer inlet. Although, a matrix is not necessary for ionization, embedding the analyte in a matrix or solvent increases the sensitivity by orders of magnitude; only femtomoles of, for example, myoglobin are necessary for observing multiply charged molecular ions using a solvent or matrix. Inlet ionization using a matrix is also a "soft" process. Fragile peptide modifications such as O-GlcNAc (Figure S1) as well as gangliosides are detected intact and with little fragmentation [53].

MALDI matrix compounds thus far tested produce $[\mathrm{M}+$ $\mathrm{H}]^{+}$ions when introduced neat into the heated mass spectrometer inlet. However, when used as a matrix with incorporated peptides or proteins as analyte, a number (e.g., SA and CHCA) produce multiply charged ions in AP-LSI, if at all, only at a high inlet temperature $\left(>400{ }^{\circ} \mathrm{C}\right.$ ) (Figures S2, $\mathrm{S} 3)$. Nevertheless, any means of introducing solid matrix/ analyte, using for example 2,5-DHAP [43], 2,5-DHB [18], 2-NPG [44] or 4,6-DNPG [44], as well as a host of other compounds as matrix materials [44], into a heated inlet linking a higher pressure region with a lower pressure region will produce mass spectra of analyte similar to ESI. These results require minimal thermal energy under low pressure conditions (vacuum assistance). For the protein ubiquitin, multiply charged ions are observed with AP-LSI and MAII using the matrix 2,5-DHAP at an inlet temperature of just $120{ }^{\circ} \mathrm{C}$ and ions are detected as low as $40{ }^{\circ} \mathrm{C}$ (Figure S4). Also in AP-LSI (and MAII), the production of up to 67 positive charges on BSA using 2-NPG as matrix was observed with only $200{ }^{\circ} \mathrm{C}$ applied to the MS inlet tube [19].

Experiments with peptides and small proteins in the UV absorbing matrix 2,5-DHAP using IR $(1064 \mathrm{~nm})$ and visible $(532 \mathrm{~nm})$ wavelength beams from a Nd:YAG laser produce AP-LSI results nearly identical to 337 or $355 \mathrm{~nm}$ UV lasers (Figures S5, S6). A number of new matrix compounds without absorbance at UV wavelengths produce multiply charged ions in MAII where a laser is not necessary. Nonaromatic conjugated and fully saturated matrices have been shown to produce multiply charged peptide ions [44]. Even solvent-free sample preparation, used initially with 


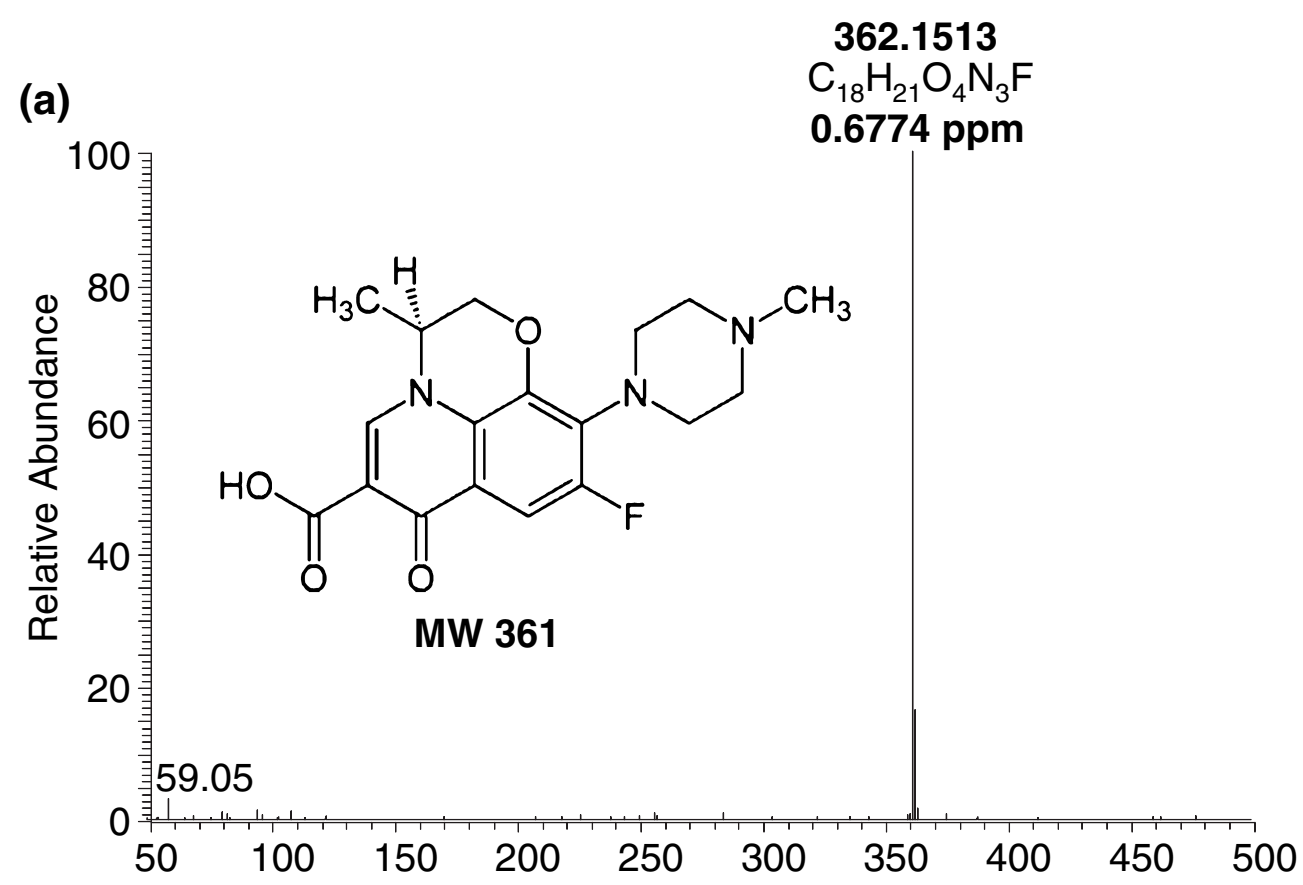

(b)

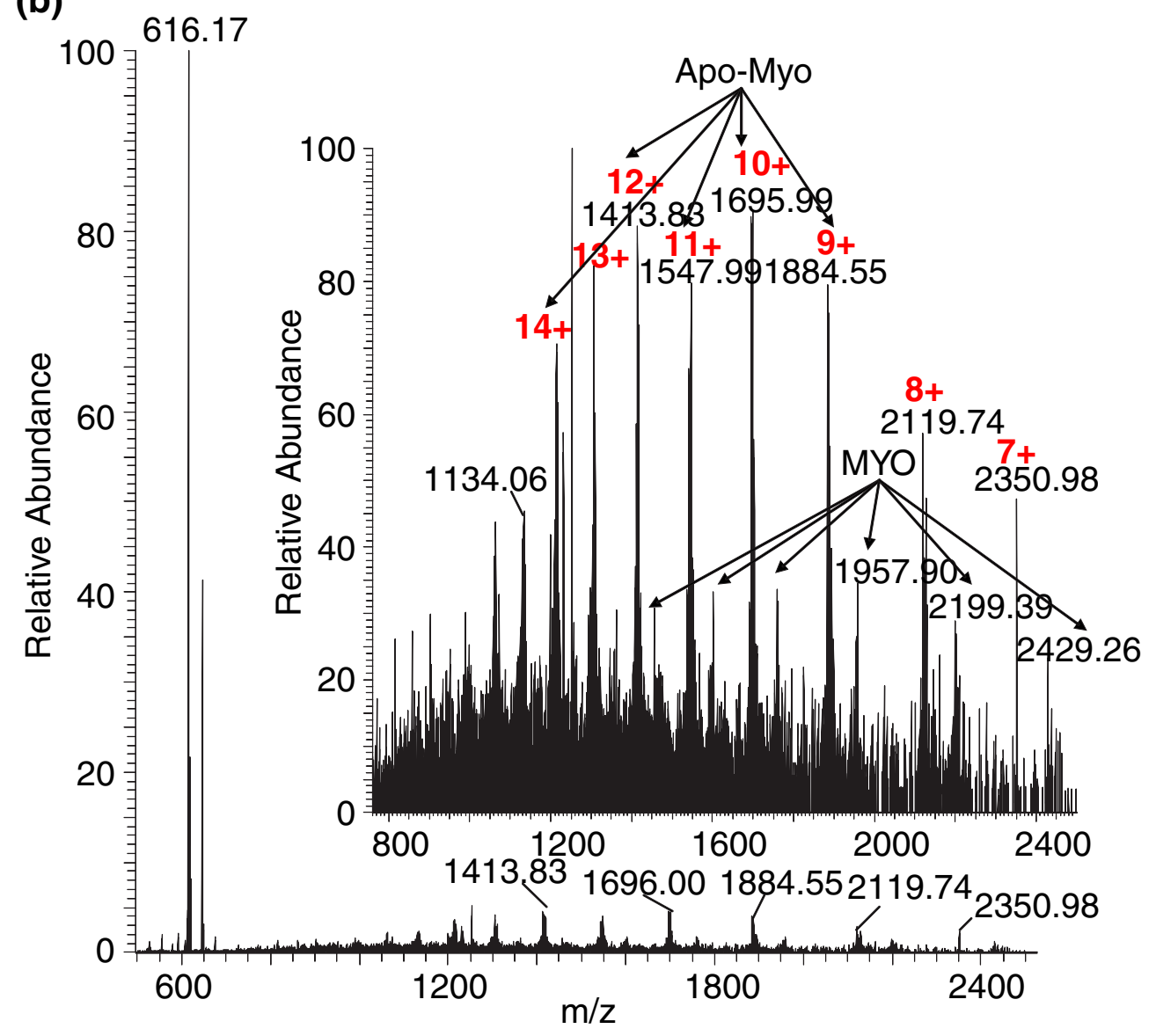

Figure 2. Mass spectra of (a) lavaquin (MW 361) powder acquired without adding a matrix and (b) pure myoglobin (MW ca. $17 \mathrm{kDa})$ powder placed directly into the heated inlet tube $\left(425^{\circ} \mathrm{C}\right)$ of a Thermo Orbitrap Exactive mass spectrometer

MALDI [54], produces highly charged ions from peptides and proteins if the matrix and analyte are sufficiently ground together to produce small particles of matrix and analyte in intimate contact [47]. Assumptions for ionization in MALDI 
prior to the 'lucky survivor' model [10] involved photoionization $[6,9,33,41]$, however, inlet ionization (Figure 3-I; Figures S2, S3) demonstrates that a laser is not necessary to produce analyte ions from matrices used in MALDI. Lasers, however, are a beneficial means of introducing sample into the inlet by ablation because the laser beam can be focused on small areas, a requirement for high resolution imaging [55].

\section{Droplet/Cluster Charging for Producing Multiply Charged Ions}

A laser is not required for producing ions from a matrix, but this does not eliminate photoionization as a possible contributor for charging clusters in LSI or MALDI. Experiments show that most matrix particles are either positively or negatively charged upon ablation at AP, but this in itself also does not imply charging by photoionization [56]. Deflecting the charged matrix particles, using electric fields as high as $800 \mathrm{~V} \mathrm{~mm}^{-1}$, before they reach the heated mass spectrometer inlet tube relative to not removing the charged particles results in little change in the observed AP-LSI mass spectrum of insulin in 2,5-DHAP. This shows that few, if any multiply charged bare ions are produced before the inlet. Additionally, had multiply charged ions been produced at AP by laser ablation prior to entering the inlet, these ions would pass through the inlet tube and to the detector of the mass spectrometer irrespective of the inlet tube temperature, just as occurs with ESI [24]. However, few multiply charged ions are observed with the inlet tube at low temperature in AP-LSI $[23,24]$ as can be seen in a temperature study from 50 to $450{ }^{\circ} \mathrm{C}$ using the matrix 2-NPG with the laser beam untenanted (Figure 3a-I, MAII, and -II, AP-LSI). In sum, these results show that in AP-LSI, photons are not responsible for multiply charged ion formation.

The nearly identical charge states produced in IP-LSI relative to AP-LSI using 2-NPG or 2,5-DHAP matrices for peptides and small proteins [42] suggests that photoionization is not responsible for multiply charged ions produced under IP conditions. The operational similarity of IP-LSI and MALDI further suggests that photoionization may also not be a strong contributor to cluster ionization in MALDI. This agrees with the observation by Niu et al. [1] that UV- and IR-MALDI produce very similar results suggesting that ionization is not the direct result of electronic excitation of the matrix by photons, but a natural consequence of the solid to gas-phase transition induced by the irradiation.

Charged molecules, especially those that are multiply charged, cannot be efficiently evaporated from a solid surface into the gas phase. The work necessary for a singly charged ion to overcome its electrostatic image charge has been calculated for an ice surface along with the probability of escape of the ion relative to the uncharged molecule [57]. This analysis demonstrates that evaporation of an ion from an ice surface is negligible relative to that of the corresponding neutral molecule (ca. $10^{-57}$ at $250 \mathrm{~K}$ ).
However, this analysis also demonstrated that the work required to separate an ion from its image charge is greatly reduced if the ions are solvated. Therefore, separation of an ion in a solvent cage from a surface is a lower energy process than removal of a bare ion. Iribarne and Thompson [31] showed that removal of a solvated ion from the surface of a highly charged droplet was facilitated by the repulsive field. Therefore, in order to observe a nonvolatile gas-phase ion it seems necessary that charged droplets must first be generated.

\section{Production of Charged Droplets or Particles without an Electric Field}

Charged droplets or solid particles are common in nature as seen in electrical storms, waterfalls, and volcanoes [58, 59], and can be induced by application of an electric field [60]. Indeed, studying formation of charged droplets/particles from boiling liquids [61] and from ice crystals has a long history [62]. However, there are numerous proposed mechanisms that can contribute to the charging of droplets/ particles [63]. Triboelectric [64], electric double layer [65], or spray [66] charging are a few possible explanations for the initial charging in inlet ionization at AP. By such mechanisms, particle or droplet contact with a surface could initiate charging (Figure S7). However, the finding that nearly equal ion abundances and charge states are produced using different materials for inlet tube construction, and the recent demonstration that laser ablation of 2,5-DHAP [42] and 2-NPG [45] under IP conditions produce highly charged ion distributions nearly identical to those seen in AP-LSI are not easily reconciled with a purely surface charging mechanism. An alternative ionization possibility for matrix charging that can operate at AP and IP is statistical charging by an explosive fracturing mechanism, including such processes as bubble bursting. Such a mechanism was described by Vestal for particle bombardment and laser ablation of surfaces [29]. He suggested that superheating a surface under vacuum conditions by an energy sudden event resulted in bubbles bursting and forming possibly a supersonic jet. Sunner et al. [67] and Kosevich et al. [68] suggested superheating below the liquid surface as a model for desorption/ionization processes in FAB. Kosevich et al. [68] compared the formation of bubbles which burst releasing analyte and charged droplets under vacuum conditions to a bubble chamber. Karas et al. proposed such a process as a possible charging mechanism in the 'lucky survivor' model for MALDI [10]. In these mechanisms, charge separation is directly initiated by the particle bombardment or laser ablation events. Statistical charging suggested by Vestal [29] and as described by Dodd [30] is expected to produce equal net positive and negative charge and produced less than 5 charges/micron diameter on water droplets. Such a mechanism would seem sufficient for processes that produce primarily singly charged ions. 
I. MAII

(a) $50^{\circ} \mathrm{C}$

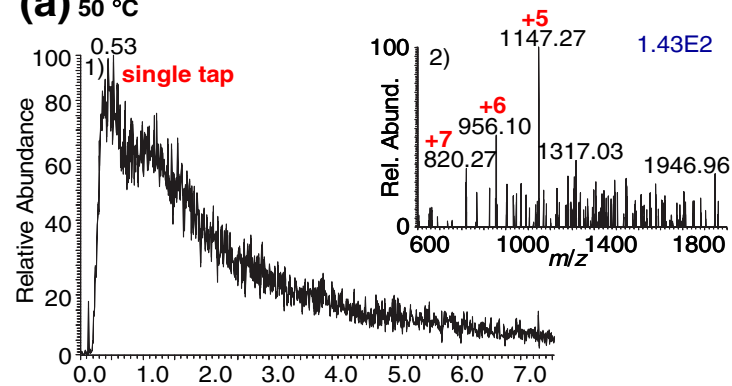

(b) $100{ }^{\circ} \mathrm{C}$

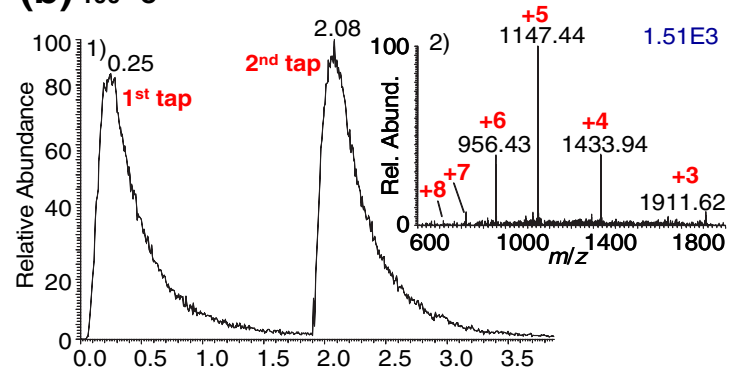

(c) $150^{\circ} \mathrm{C}$

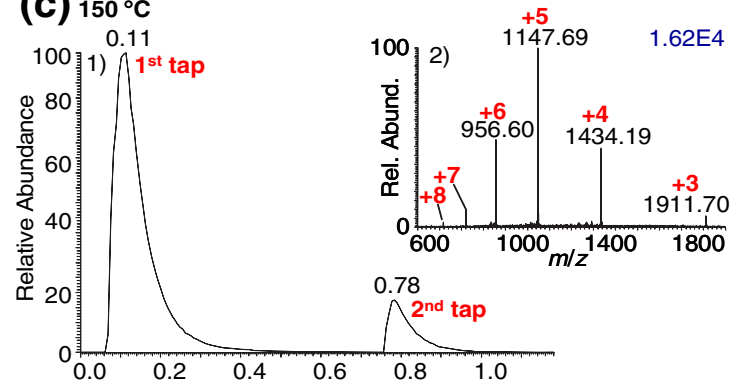

(d) $300^{\circ} \mathrm{C}$
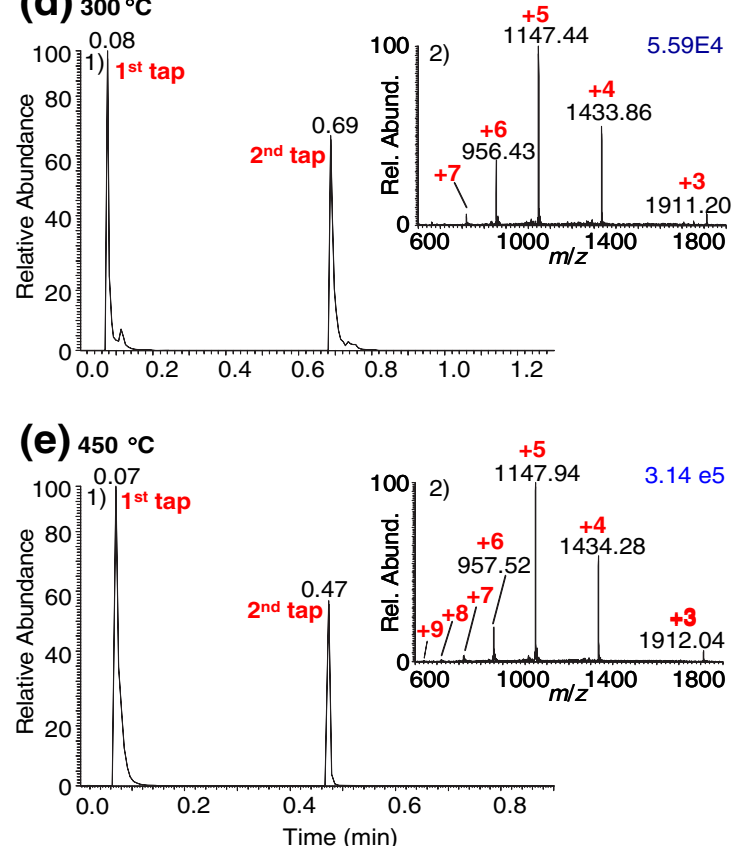

II. LSII

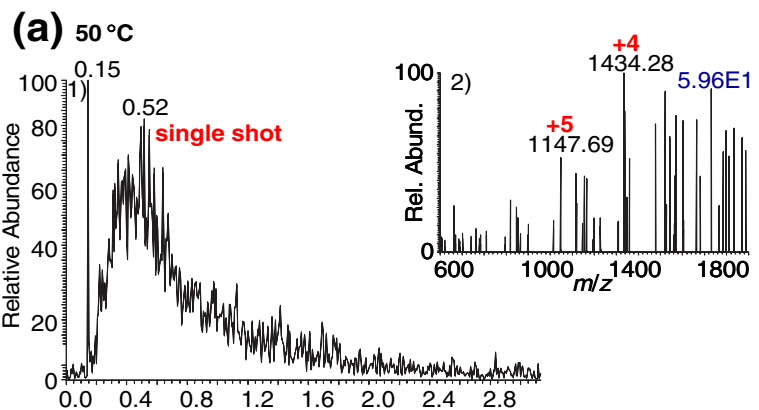

(b) $100^{\circ} \mathrm{C}$

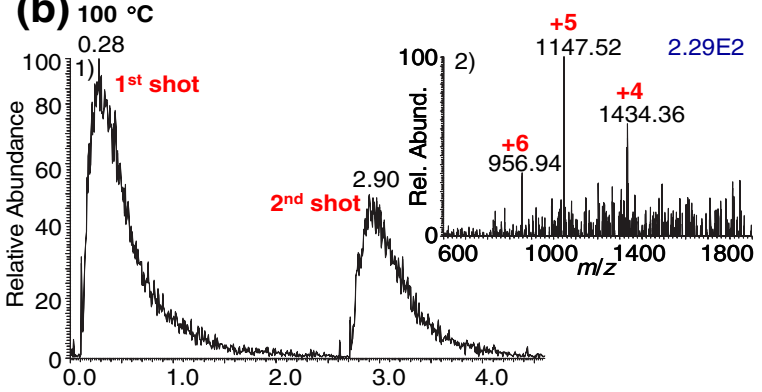

(c) $150^{\circ} \mathrm{C}$

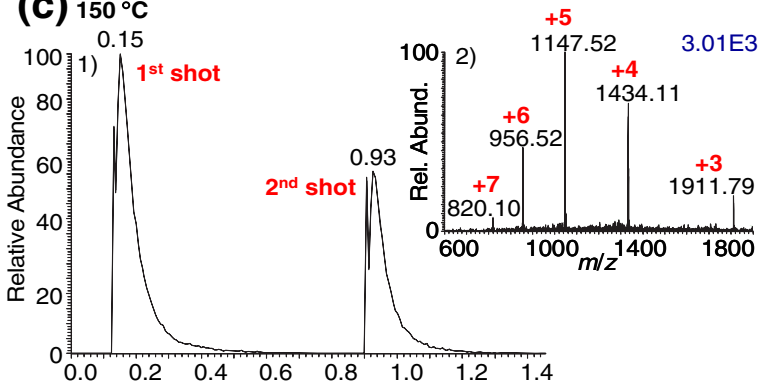

(d) $300^{\circ} \mathrm{C}$

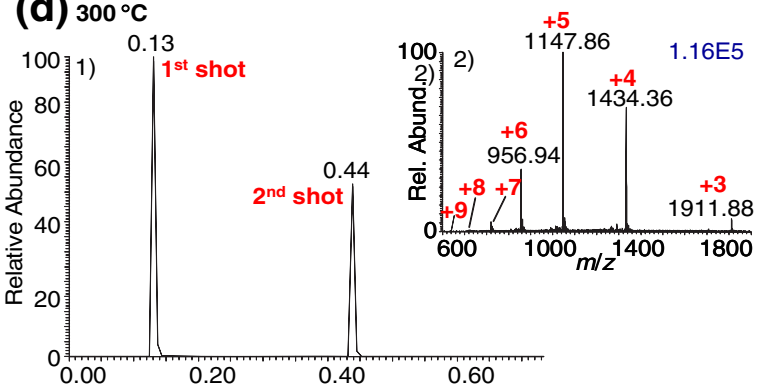

(e) $450{ }^{\circ} \mathrm{C}$

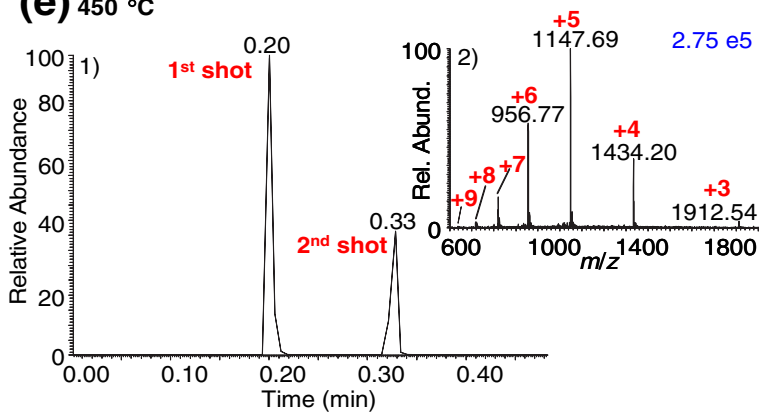

Figure 3. Temperature study of 2-NPG matrix using angiotensin I (MW 1295) (I) MAll and (II) AP-LSI at (a) $50{ }^{\circ} \mathrm{C}$, (b) $100^{\circ} \mathrm{C}$, (c) $150^{\circ} \mathrm{C}$, (d) $300{ }^{\circ} \mathrm{C}$, and (e) $450^{\circ} \mathrm{C}$ showing the (1) ion current chromatogram and (2) inset mass spectra acquired using a Thermo LTQ-Velos mass spectrometer 
Observation of highly charged ions by a mechanism similar to ESI requires formation of droplets with many more charges than expected from a statistical process. In inlet ionization, this must be accomplished without a voltage. Such a process is obviously possible as it is now known that water droplets in the upper and lower layers of clouds have hundreds of positive and negative charges, respectively [63, 69]. Zilch et al. [70] observed that when charged droplets were passed through a tube linking AP to vacuum, the charge on the droplets increased. This was attributed to aerodynamic breakup by a postulated nonstatistical 'bag' mechanism. As many as 100,000 charges were observed on micron size droplets, initially produced by sonic spray ionization, after passing through the capillary. The 'bag' mechanism involves ions of one charge collecting preferentially at the liquid-gas interface. Breakup of a thin liquid surface, such as occurs in a bubble, will carry more of the surface charge, negative in the case of water, and the positive charge resides with the thicker edge of the 'bag'. Another way to view this is that an electric double layer forms at a liquid-solid or liquid-gas interface. This was the explanation provided by Iribarne and Thompson [31] for droplet charging in a sonic spray type experiment. Breakup of the electric double layer was originally postulated to produce droplets with few charges by a nonstatistical process [71]. However, in rapid bubble formation, a shearing of the electric double layer could segregate charge due to inertia of the charge carriers. Bubble bursting will produce very small droplets with an excess of the surface charge. while the larger remaining droplet has the opposite charge. It is likely that this mechanism only works if the entire process is faster than the diffusion processes that would restore charge equilibrium.

In AP-LSI, the matrix particles are heated as they traverse from AP to the first vacuum region of the mass analyzer. The rapid movement of heated liquid droplets through a pressure drop region naturally leads to a superheated state which may be aided by collisions of the matrix/analyte droplet/particle with the hot wall surface. Under vacuum conditions used in MALDI, it is also possible for the ablated molten matrix/analyte droplets to experience superheating in the expanding plume while traversing the pressure gradient from solid to vacuum similar to that described for AP-LSI. Only a nucleation event [68] is required to violently release the excess energy in bubble formation and bursting leading to highly charged droplets.

Segregation of charge of one sign at a surface interface (e.g., air-water, water-ice) is common, and it would appear that any means of rapid fracturing that results in removal of more of the surface relative to the bulk could result in drops or particles of opposite charge being generated. For example, ice particles have been implicated in the charging of water droplets in thunderclouds by such a mechanism [63]. In collisions between ice particles of different size or temperature, or in splintering of the ice surface, smaller droplets/particles carry away the surface charge, leaving the larger droplet with excess of the opposite charge. Growing ice particles have a negative surface charge and subliming ice particles more positive surface charge. Thus, loss of some of the surface charge on a subliming particle will result in the particle acquiring a net negative charge. The segregation of charge is related to the relative diffusion rates of $\mathrm{H}^{+}$and $\mathrm{OH}^{-}$.

The effect of temperature on the surface charge of droplets/particles can readily be observed using a simple home constructed apparatus having a metal inlet tube, similar to that used in AP-LSI, with the entrance end at AP and the exit end in a low pressure region created by a rotary pump. When water is introduced into the heated inlet tube, positive charge is measured downstream in the low pressure region using an electrometer, and the inlet tube acquires a negative charge. The charge acquired by the inlet tube is associated with the droplet surface, because the surface-air interface in water droplets is negatively charged. However, with ice, the surface charge will be positive under subliming conditions [63]. By cooling the inlet with dry ice, a charge reversal occurs when water is introduced into the inlet so that a negative charge is observed downstream and the inlet tube charges positive. Charges are also generated by numerous other solvents and solid matrix compounds passed through a heated inlet. We have also observed a reversal of charge at different temperatures with some solid matrix compounds. One explanation of the measured charge is that the droplet surface gives up its charge on contact with the metal tube; however, because smaller droplets/particles carry away the surface charge, another explanation is that these smaller particles diffuse to the inlet tube walls faster than the larger droplet/particles. In thunderclouds, and in the work described by Zilch et al. [70], large particles carry the opposite charge of small particles/droplets, and turbulent conditions provide a mechanism for the charges to diffuse away from each other reducing ion-ion recombination loses.

Other means can be considered for providing charge separation. A sudden release of gas during explosive matrix decomposition can be envisioned initiated by the laser event as first proposed Zubarev et al. [72] and later also demonstrated by Hakansson et al. [73] for matrix-assisted plasma desorption mass spectrometry using nitro-containing compounds. Decomposition reactions [74-76], for example decarboxylation, are well known and have been studied as a function of temperature with DHB isomer matrices [76] and discussed in MALDI [5]. Ablated matrix droplets in which small neutral molecules are released by thermal decomposition of the matrix could produce violent bubble formation on expansion of these gases as the molten droplets rapidly move from a high to low pressure regime of the expanding plume for IP-LSI, and in the heated inlet tube for AP-LSI. In support of this as a possible mechanism for assisting droplet charging is the recent findings by Trimpin et al. [44], demonstrating that the thermally labile polymer azo initiator $\mathrm{NC}\left(\mathrm{CH}_{3}\right)_{2} \mathrm{C}-\mathrm{N}=\mathrm{N}-\mathrm{C}\left(\mathrm{CH}_{3}\right)_{2} \mathrm{CN}$ produces excellent abundance multiply charged analyte ions using MAII if the 
matrix/analyte solution is acidified before drying. With this matrix, release of $\mathrm{N}_{2}$ could result in dislodging surface droplets/particles as the gas expands under low pressure conditions. Acid conditions may be required for matrices which have no acidic hydrogen atoms. A similar azo structure, but containing a carboxylic acid group works well as a matrix without adding acid [44]. In summary, production of highly charged droplets or particles is a process that can readily occur under a variety of conditions and the utility of charged solvent droplets for producing ions in mass spectrometry is well documented.

Observations [37, 77] and modeling [6, 34, 36, 78, 79] showing that laser ablation under vacuum conditions produces droplets/clusters, and the observation of highly charged ions from a laser ablation process at IP are highly suggestive of ionization initiated from charged matrix droplets similar to the ionization mechanism proposed for AP-LSI. Highly charged ions are only observed under IP condition on the MALDI source of a SYNAPT G2 when there is some extraction voltage (as little as $1 \mathrm{~V}$ ) between the sample plate and the first lens suggesting that ionization, at least of the clusters, occurs before the extraction lens. Under typical MALDI-TOF conditions, a similar process to laser ablation at IP could also occur. Thus, formation of highly charged matrix droplets/clusters might be expected to be produced under MALDI-TOF conditions as proposed in the initial 'lucky survivor' paper [10].

Studies using delayed extraction and based on observation of singly charged ions in a MALDI-TOF instrument suggest no charge exists on ion forming clusters when they first leave the ablated solid surface under vacuum conditions, but charges appear on the matrix clusters at about $200 \mathrm{~ns}$ and continue to increase with time in the range 200 $800 \mathrm{~ns}$ after the ablation event $[9,41]$. This delayed cluster ionization is not likely to result from photoionization and was interpreted as loss of electrons from matrix clusters. Kinsel et al. [33] also suggested a delayed ionization process. Vacuum assisted superheating and fracturing by, for example, bubble formation on release of the excess energy would explain the preponderance of initial ion formation being a delayed process.

\section{Bare Ion Formation in LSI and MALDI}

An important observation relative to the MALDI mechanism is that a number of matrix compounds produce highly charged ions with AP-LSI [44], but thus far, few matrices produce in abundance highly charged ions under vacuum conditions. For example, 2,5-DHB produces multiply charged ions in very low abundance under IP-LSI conditions with the SYNAPT G2 MALDI source, but 2,5-DHAP and 2NPG matrices produce abundant highly charged ESI-like ions under vacuum conditions $[42,45]$. In AP-LSI, the inlet tube temperature necessary to produce multiply charged ions is 2 -NPG $<2,5$-DHAP $<2,5$-DHB $<$ CHCA, which is also the order these compounds vaporize or sublime with slowly increasing temperature at $\mathrm{AP}$ or prolonged exposure to vacuum conditions [44]. On the LTQ Velos mass spectrometer, CHCA produces multiply charged ions using AP-LSI and MAII only at the highest inlet temperature $\left(>425{ }^{\circ} \mathrm{C}\right)$ (Figure S3.I), whereas 2-NPG requires $<200{ }^{\circ} \mathrm{C}$ (Figure 3). An interpretation of these results is that charged matrix droplets/particles are produced by laser ablation of any of the above matrix compounds and that release of highly charged analyte ions in AP-LSI is influenced by the volatility of the matrix and the heat applied in the sub-AP environment because of the critical need of desolvation.

With AP-LSI, heat provided by the inlet tube along with low pressure conditions are believed to be sufficient to provide both the initial matrix droplet charging and the energy and time necessary to desolvate these droplets and release of multiply charged ions in an ESI-like process. There is also evidence that collision with a surface and possibly fields (e.g., radio frequency (rf)), in combination with gas-phase collisions can enhance the desolvation of charged clusters [42, 44]. For example, increased ion abundance of multiply charged ions is obtained at lower inlet temperature by placing an obstruction at the end of the mass spectrometer inlet tube so that charged clusters, not completely desolvated on exiting the inlet tube, collide with the obstruction (Figure S8). We have shown a similar result with sonic spray ionization where a surface obstruction greatly increased the observed ion abundances [24, 80]. Recent molecular dynamics simulations also suggests the importance of collisions for desolvation in ESI [81]. With typical AP and IP MALDI instruments, desolvation of the matrix with release of bare analyte ions can occur anywhere before the mass analyzer without affecting the mass measurement accuracy. Thus, desolvation could be enhanced within rf fields or, for the SYNAPT G2, the traveling wave device within the instrument $[42,82]$. This is not the case for MALDI-TOF instruments where bare ion formation after acceleration will not be correctly mass analyzed.

\section{Time and Space Consideration}

MALDI has primarily been defined by the ions observed at the detector of a mass spectrometer. The detected ions, however, are in part determined by the instrumentation and instrumental conditions. Mechanisms for MALDI ionization are based primarily on data from MALDI-TOF mass analyzers, but observation of bare analyte ions from a droplet/cluster using a MALDI-TOF instrument requires not only that the droplets/clusters become charged in a prompt process, but that the bare charged analyte ions are released from the droplets/clusters in an extremely short time (typically $<1 \mu \mathrm{s}$ for delayed extraction) [4]. Only analyte ions which have shed the matrix molecules prior to high voltage acceleration will be detected at the correct flight time for the analyte or matrix molecular mass. Accelerated clusters will reach the linear detector based on the mass and charge acted on by the acceleration voltage irrespective 
of the cluster mass on reaching the detector. Because there are numerous possibilities for the mass of and the charge on droplets and clusters, they will contribute to the background as previously reported [35].

The desolvation necessary for emission of bare multiply charged ions from the charged droplets is likely the slow step. Chang et al. [4] pointed out that desolvation of water from charged droplets in ESI occurs at AP and over centimeter distance with applied heat, and yet in MALDI, desolvation of solid matrices must occur within about $1 \mu \mathrm{s}$, which is orders of magnitude less time than required in ESI. Measurement of cluster sizes under MALDI conditions confirmed that clusters as large as ca. $250 \mathrm{~nm}\left(50 \times 10^{6}\right.$ matrix molecules) are formed [4]. Under MALDI-TOF conditions, the energy required to desolvate even much smaller clusters is not available [5].

The observation of stable multiply charged bare ions in MALDI-TOF and IP-MALDI instruments using matrices common to LSI, and the requirement that such ions must be produced directly from the solid or liquid state, as noted above, is best explained by charged matrix droplets/clusters being produced in MALDI upon laser ablation. However, in MALDI-TOF instruments, matrix evaporation limits the bare ions that can be produced from charged droplets within the time/distance before acceleration. Factors affecting the ability to release bare ions from ablated matrix based on the need for desolvation are the size of the ablated droplet/ clusters, their internal energy upon laser ablation, the volatility or sublimation potential of the matrix, and the time before acceleration.

Interestingly, the most volatile, 2-NPG, and least volatile, CHCA [44], of the above discussed matrices, produced the highest charge states for small proteins [45]. It is well known that CHCA produces multiply charged ions from proteins in MALDI [1], but relative to the matrix 2-NPG, the average charge state is lower and the energy imparted to the ions is higher. With CHCA, the multiply charged ions undergo metastable fragmentation in the linear flight tube making higher resolution reflectron measurements of multiply charged ions problematic [28, 45, 46]. On the other hand, the multiply charged ions produced with 2-NPG, as expected for ions formed by the LSI mechanism, are stable and pass through the reflectron as shown for carbonic anhydrase in Figure 4. CHCA also produces highly charged ions from small proteins in low abundances under IP conditions on the SYNAPT G2 even though this matrix requires $>425{ }^{\circ} \mathrm{C}$ to produce multiply charged ions in MAII (Figure S2.I and S3.I), or AP-LSI.

The two-step photochemical model does not readily explain higher charge states with CHCA, and unless CHCA produces more energetic and/or smaller charged clusters during the ablation process so that desolvation occurs in spite of its low volatility, it is also hard to explain formation of multiply charged ions by the mechanism proposed for LSI. Musapelo and Murray [40] recently reported that CHCA produces a bimodal distribution of particles upon

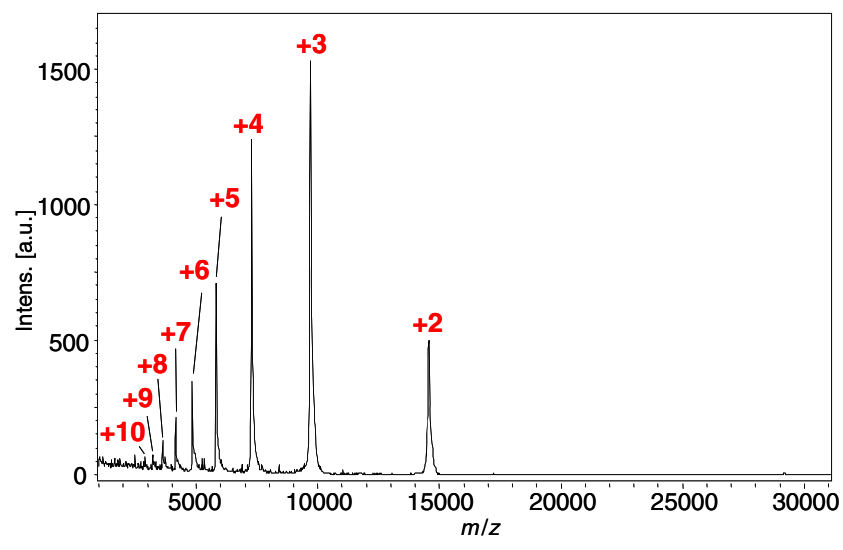

Figure 4. Mass spectra of carbonic anhydrase (MW $29 \mathrm{kDa})$ using 2-NPG as matrix acquired in reflectron mode using a Bruker MALDI-TOF/TOF mass spectrometer using instrument manufacturer recommended settings

laser ablation at AP, one with a size distribution centered at about $20 \mathrm{~nm}$, very near the minimum size they could measure. Because other matrices studied also produce particles in this size range, for CHCA to produce multiply charged ions by matrix evaporation from charged clusters, while the more volatile matrices are more limited in this regard, seems to require small ablated clusters hold more charge with CHCA, or the clusters are hot relative to clusters produced from other matrix compounds. A hotter matrix cluster is supported by work of Allwood et al. [83] showing that CHCA has the highest absorption coefficient of several MALDI matrices when measured as thin films, and by Bae et al. [84] showing that CHCA not only has a higher absorption coefficient, but is hotter than SA, DHB, or glycerol as matrix in both the early and late MALDI laser ablation plume. The observation that the multiply charged protein ions produced using CHCA as matrix fragment in the linear flight tube supports a higher energy ion formation process. Thus, CHCA may be a case where the internal energy of small clusters is sufficiently high to rapidly evaporate the matrix releasing hot multiply charged ions.

An intriguing observation related to multiply charged ions formation is that two charge state distributions are observed under LSI conditions in an IP-MALDI source using 2-NPG as matrix (Figure 5a), but 2,5-DHAP produces solely the high charge state distribution similar to AP-LSI [85]. 2,5-DHAP does not produce the lower charge state distribution, observed with 2-NPG, in a MALDI-TOF instrument. Thus, with 2-NPG using IP-LSI, one distribution reflects the high charge states observed with AP-LSI (Figure 5b), and the other, the lower charge states observed in the MALDI-TOF instrument [45]. Therefore, two distinct modes appear to exist for producing multiply charged ions using 2-NPG as matrix: one is dominant at AP and the other at LP and both operate under IP conditions. The low charge state distribution can be promoted using 2-NPG under IP condition by increasing the laser power (Figure S9). This lower charge state distribution observed under LP conditions 

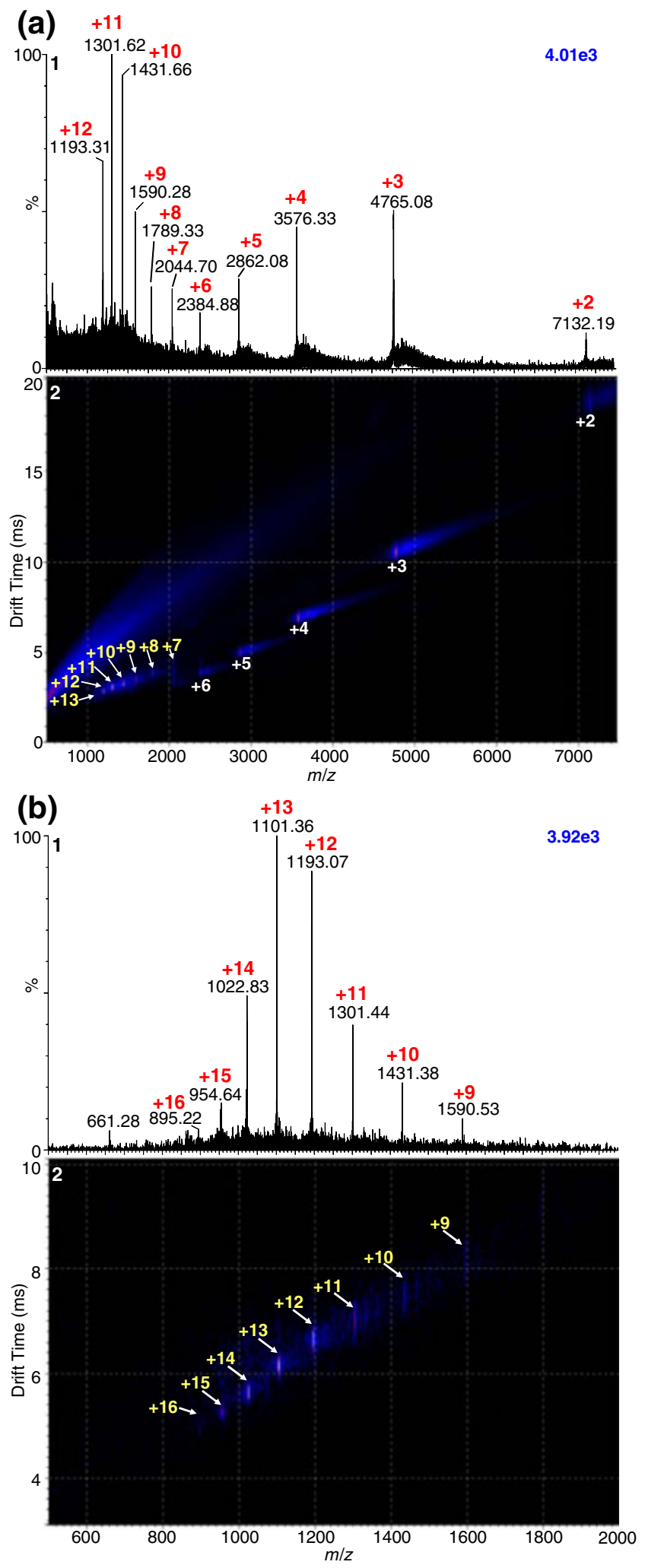

Figure 5. (1) Mass spectra and (2) 2-dimensional plot of $\mathrm{m} / \mathrm{z}$ vs. drift time of lysozyme (MW $14.3 \mathrm{kDa}$ ) acquired using (a) IP-LSI and (b) AP-LSI with 2-NPG matrix on a Waters SYNAPT G2 mass spectrometer

fits a model where ions are only produced from charged clusters sufficiently small, and thus holding few charges, to evaporate the matrix in the time domain of MALDI-TOF. Thus, the fact that the charge state distribution observed with 2-NPG in MALDI-TOF is high relative to charge states produced with other matrix compounds suggests that the charged clusters from which the bare ions are produced are larger and have more charges than with other MALDI matrices. Therefore, the energy imparted to 2-NPG by the laser, and 2-NPG's propensity to rapidly evaporate/sublime, allows somewhat larger clusters holding more charges [29] to evaporate in the MALDI-TOF timeframe releasing a higher abundance of multiply charged ions. A bimodal distribution of cluster sizes, as noted for other matrix compounds [40], would allow the smaller clusters to produce ions with MALDI-TOF instruments, and both the smaller and a larger cluster/droplet distributions to produce bare ions under the longer time domain available in instruments with IP-MALDI sources. If this is the case, the observation under IP conditions that the lower charge state is favored at high laser power might suggest smaller clusters are favored with increased laser fluence.

Assuming that the formation of highly charged stable ions using laser ablation under IP and LP conditions [42, 45] occurs by the ion evaporation [31] or charged residue [48] models proposed for ESI necessitates evaporation/sublimation of matrix from charged matrix droplets/clusters. Under IP and LP conditions, using commercial instruments, no external thermal energy other than from the laser is supplied. The observation, as well as the expectation, is that matrix compounds that absorb the laser energy, converting it to thermal energy, and readily evaporate or sublime will produce the more highly charged stable ions under vacuum conditions. Thus, the inability to evaporate or sublime most matrix compounds from highly charged droplets with the energy supplied by the laser and in the short time and distance available in MALDI-TOF before ion acceleration and/or evaporative cooling can explain why highly charged ions are generally not observed in abundance in MALDITOF instruments even though highly charged droplets of matrix are formed. Evaporative, or expansion, cooling is expected to quickly produce solid particles even under IP conditions, so that desolvation by sublimation of matrix at longer times in the instrument might result in ion emission from solid particles. As a reference, the temperature of DHB, whose melting point is ca. $475^{\circ} \mathrm{K}$, was found to be in the range $423-589{ }^{\circ} \mathrm{K}$ in the late plume in MALDI-TOF [84].

The above discussion suggests that the study of singly charged ions produced in MALDI-TOF instruments provides a myopic view of ion formation in the laser ablation process. Given time, thermal energy, and a sufficiently volatile matrix, multiply charged ions are expected from charged ablated droplets/clusters. Their observation in MALDI-TOF [45] and IP-MALDI [42] instruments as well as in AP-LSI $[17,47]$ clearly demonstrates that charged matrix/analyte droplets produce ions under these conditions. This analysis strongly suggests that the sensitivity of vacuum MALDI can be enhanced by finding a means of desolvating the 
charged matrix droplets/particles, using matrix compounds that desolvate in the MALDI-TOF timeframe, or by extending the time before ion acceleration in MALDITOF instruments.

\section{Formation of Singly Charged Ions in MALDI from Clusters}

Singly charged ions are a natural consequence of the twostep photochemical model, but because ESI and LSI produce multiply charged ions of peptides and proteins from droplets, it suggests that formation of singly charged ions directly from liquid or solid droplets/clusters requires special conditions not achieved with these soft ionization processes [5]. The requirements necessary to form singly charged ions differentiates various cluster mechanisms $[2-4,6,8,10,34$, 41]. Kinsel et al. [33] first suggested the existence of two mechanisms for ion formation in MALDI: a prompt ionization process in which protonated matrix and analyte ions are formed, and a delayed process that best fits a cluster mechanism with delayed analyte ionization. Fournier et al. $[9,41]$ suggested two distinct ionization mechanisms, the most important of which produces ions from clusters. As noted above, MALDI is a prompt ionization process occurring within microns of the matrix surface and a microsecond of the ablation event $[7,86]$ and, thus, even the delayed ionization is prompt relative to ionization in IPor AP-LSI where the bare ions may be formed many millimeters or centimeters from the source of ablation by desolvation of highly charged droplets.

These observations along with the recent experimental data obtained by Jaskolla and Karas [2], showing both the gas-phase and cluster mechanisms are active in MALDI, at least for the small molecules studied, suggest that laser ablation at IP produces a mix of ablated materials ranging from vaporized molecules to matrix clusters, droplets, and particles having a range of sizes, charges, and energies. Different matrices and conditions are expected to produce different mixes of these ablated materials. The early laser ablated plume is hot (ca. $1000^{\circ} \mathrm{K}$ ) [6] and may contain ions from photoionization as well as from charged clusters directly produced in the ablation process, and is likely where most of the gas-phase ionization occurs. This is also where rapid dissociation of protonated labile species is observed [84]. Singly charged ions produced from charged clusters would likely be the delayed process occurring later in the plume. Thus, a mechanism for selection of singlycharged ion production from charged clusters is needed.

In Vestal's 1983 unified mechanism paper, singly charged ions are proposed to be field-evaporated from charged droplets [29]. However, in the meantime, ESI, and other techniques known to involve charged droplets, have shown that producing singly charged ions from these methods is problematic for larger molecules with multiple basic sites. Inlet ionization shows this to also be true for solid solvents (matrices). In fact, with even a small protein falling within the mass range of common AP ionization instruments, singly charged ions are typically not observed by these methods.

Fenn attempted to explain the charge distribution in ESI [87] beginning with assumptions from Iribarne and Thompson's ion evaporation model [31]. Ion evaporation and the charge distribution model are well explained by Fenn, and only a brief simplifying synopsis is presented here. Lifting ions from a charged droplet requires work to overcome an activation barrier of which a significant fraction is related to surface tension. The surface tension is increased as the droplet surface area increases upon lift-off of the ion and associated solvent molecules. The lift-off activation barrier is lowered by the field acting on the analyte charge(s) as it is being removed from the like-charged droplet. Any difference between the energy supplied by charge repulsion and the energy required to surmount the activation barrier must be provided by the thermal energy associated with the droplet. A simplification is that the higher the charge on the analyte and the smaller the analyte ion size, including any accompanying solvent molecules, the lower the thermal energy required for desorption. This also means that a singly charged protein ion will require a great deal of thermal energy to field evaporate from a surface.

According to Fenn's model, charges reside in equidistant potential wells on the droplet surface. Ionization of analyte, possibly by neutralization of one of its anions, anchors the analyte charge center to the potential well. As the droplet evaporates, the charges move closer together allowing them to interact with more basic sites on the analyte molecule. The number of charges that can be attached to the analyte ion as it leaves the droplet surface is determined by the number of surface charges the basic sites of the analyte molecule spans, which is determined by the charge density at the droplet surface. Thus, for larger molecules with multiple basic sites such as peptides and proteins, in ESI, there will be several charges on the molecule before the field strength at the droplet surface is sufficiently high to field evaporate the analyte ion. In accordance with the above discussion, higher energy imparted into a droplet will allow field evaporation at lower repulsive fields and, thus, at lower droplet charge density, favoring lower charge state ions. Under IP laser ablation conditions, lower laser power favors multiply charged ions and higher laser power singly charged ions (Figure 1), which is qualitatively in agreement with this model, as are observations that in inlet ionization higher inlet temperature favors singly over multiply charged ions $[17,23,24]$.

Another proposal to explain singly charged ions in MALDI was provided by Alves et al. [3] studying low charge state multiply charged ions. These authors suggested two mechanisms for formation of ions from charged clusters: (1) a low energy desolvation similar to ESI involving loss of neutral matrix molecules and resulting in a densely charged surface where field evaporation of multiply charged ions occurs, and (2) a high energy process in which matrix ions 
are given sufficient thermal energy to field evaporate, thus lowering the cluster charge state, and creating bare singly charged analyte ions. In this second scenario, thermal energy is provided to lift matrix ions over the higher activation barrier required for an ion to leave the surface. With AP-LSI, MAII, and SAII with relatively low inlet temperature, and in ESI, only the low energy pathway is operative, while in laser ablation there is sufficient energy for the higher energy process to be operative.

A reason highly charged ions are not observed in MALDI may be partly because of selection in the MALDI instrumentation. The Alves and Fenn models can be operative, but for larger charged clusters, expansion cooling should reduce the energy within the droplet so that a crossover occurs to the lower energy process thus producing multiply charged ions. Here we propose that highly charged ions are not observed because only small clusters will have time in MALDI-TOF to completely evaporate matrix molecules, and in addition, only a low number of charges can be carried by small clusters. Thus, the limited time available in MALDITOF for analyte ion formation is an alternative explanation to the 'lucky survivor' model for observation of singly and low charge state ions from charged clusters $[2,8,10]$. Of course, the processes occurring in the laser plume are complex and this represents just one possible ionization mechanism, but one that may be important in ionization of nonvolatile compounds. As noted above, MALDI is not the only ionization method for which singly charged ions are proposed to be formed from a charged droplet or cluster [68]. Vestal's unified ionization model suggests that charged droplets are responsible for ionization of nonvolatile compounds in a variety of ionization methods which produce substantial abundances of singly charged ions [29].

\section{Creating Higher Charge States by Laser Ablation in a MALDI-TOF MS}

MALDI production of singly charged ions is a higher energy process, relative to, for example, ESI, operating very near the matrix surface and has been well studied $[2-11,22,27$, $28,33-36,41,77-79,86,88,89]$. In any cluster ionization model, larger charged clusters or droplets produced in the initial ablation need to evaporate notably more matrix volume [4] to reach a state in which the bare analyte ions are produced. Larger droplets can also carry more charge. For more volatile matrices, such as 2-NPG, evaporation/ sublimation of neutral matrix may be sufficiently rapid that small droplets, carrying intermediate charge, produce multiply charged ions within the time constraints of MALDITOF mass spectrometer ion sources. However, given time and energy for desolvation, higher charge state ions are expected to be observed from larger highly charged droplets by an ESI-like mechanism. The time constraints of MALDI-TOF instruments are not applicable in mass spectrometers used with IP-MALDI, and, for the more volatile matrices, large clusters and droplets with high charge can evaporate/sublime over the longer available time before mass measurement.

In MALDI, the ionization yield is $10^{-3}$ to $10^{-7}$ [90] and most of the analyte resides in the ablated material, especially larger droplets. Nevertheless, vacuum MALDI is a highly sensitive technique because of high ion transmission in MALDI-TOF instruments. Improved desolvation of the charged clusters or producing more small charged clusters would provide a way to enhance sensitivity. Providing the enthalpy necessary for desolvation through a thermal process in vacuum is problematic [91]. However, innovative means of desolvation, such as collisions with surfaces or use of $\mathrm{rf}$ fields under low pressure conditions is expected to provide enhanced desolvation and thus sensitivity. This scenario also predicts that discovering matrix materials that readily evaporate or sublime in vacuum, but can be made to remain solid or liquid sufficiently long for analysis under low pressure conditions will increase the number of charges observed under vacuum laser ablation conditions and, if efficient, improve sensitivity through higher ionization yield. To this end, binary matrix mixtures are being explored [44, 92]. It has been observed that as little as $10 \% 2-\mathrm{NPG}$ in SA, a MALDI matrix that produces almost exclusively singly charged ions, gives a mass spectrum under IP laser ablation conditions in which small proteins have charge state distributions very similar to pure 2-NPG as matrix (Figure S3 IV E). It might be expected that a matrix too volatile to be used directly in IP or LP-MALDI may survive the vacuum conditions embedded in a less volatile matrix. Adding $10 \%$ 2-NPG to SA extends the analysis time in vacuum relative to pure 2-NPG which is important in tissue imaging.

\section{Caveats}

There are experimental observations that are not readily reconciled with the proposed mechanism, and these are discussed in the Supplemental Section. Also, the finding that charged clusters are available for ion production in MALDI does not rule out other potential mechanisms contributing to ionization. Finally, with our current state of knowledge, we can fall back on the quote attributed to James Watson that "no model should account for all the facts, since some data will be misleading, if not plain wrong" [93].

\section{Conclusion}

Vestal's 1983 unified ionization model [29] appears to explain in broad strokes not only the ionization processes available at the time for nonvolatile compounds, but also aspects of MALDI and inlet ionization. A charged droplet mechanism for ion formation was introduced to explain formation of singly and low charge state ions, but the role of charged droplets/clusters in producing multiply charged ions of nonvolatile compounds is now well established. Highly charged droplets appear to be the source of analyte ionization in ESI and inlet ionization embracing LSI, MAII, and SAII. A role of the matrix/solvent 
in inlet ionization and, by analogy, MALDI, may be the ability to aid segregation of opposite charges on droplet/particle surfaces vs. the bulk through ion solvation [44]. Energy sudden fracturing of the surface, by for example bubble formation and bursting, results in small droplets/particles carrying more surface charge and the larger droplet having the opposite charge. Ionization occurring from liquid matrix/analyte droplets may explain why prior incorporation of analyte into the matrix crystal is helpful in LSI and MALDI, but is not necessary, as small analyte particles closely associated with matrix can dissolve in droplets of molten matrix acting as a solvent. This would explain why in solvent-free matrix preparation, longer grinding times that produce smaller particles of closely associated analyte and matrix is required for ionization of higher molecular weight and more difficult to dissolve compounds $[18,47]$.

A major problem in proposing a charged droplet mechanism, however, is the need to explain formation of singly and multiply charged analyte ions. Indications point to formation of singly charged ions requiring a higher energy primary ionization process, at least for peptides and proteins, than formation of the respective multiply charged ions. Here we propose that the charge states observed are related to the energy imparted into the droplet/cluster, the size of charged clusters produced, and the time available for desolvation of the charged droplet/clusters before mass analysis. Thus, processes with higher energy primary ionization and less time for desolvation favor singly charged ions. Alternatively, lower energy imparted in the primary process and longer time and more volatile matrices for desolvation favors producing more highly charged analyte ions from larger more highly charged clusters. In laser ablation, the morphology of the matrix, its boiling point or propensity to sublime, its conversion of laser energy into thermal energy, and the laser fluence are factors that affect the above critical parameters [44]. A predictive outcome for vacuum laser ablation is that providing conditions favorable for desolvation of the larger, more highly charged matrix/analyte droplets will result in the observation of increased abundances of highly charged ions. Nature teaches us that formation of charged droplets is a common occurrence and no doubt other means will be discovered for their production in mass spectrometry. Ion transmission suggests that finding ways to efficiently create and desolvate the charged droplets under vacuum conditions will lead to increased sensitivity.

\section{Acknowledgment}

The authors are grateful to Dr. Alexander Makarov (Thermo Corporation, Bremen, Germany) for useful discussions on delayed ionization in inlet ionization, Timothy P. Enright (University of the Sciences) for discussion on droplet ionization mechanisms, Andrew Whatley (Waters, Manchester, UK) for providing the laser power at varying settings on the SYNAPT G2, Professor Arthur Suits and Lu Yan (Wayne State University) for access and assistance with IR and visible lasers, Professor Donald Hunt and Dr. Philip Compton (University of Virginia) for access to the LTQ-ETD mass spectrometer and the donation of the OGlcNAc sample, Professor Ken Mackie and Dr. James Wager-Miller (Indiana University) for providing mouse brain sections, and Mr. Andrew McEwen for insightful discussions related to the terminology of vacuum assisted ionization. This work was funded by Wayne State University (Rumble Fellow to E.D.I. and B.W., Schaap Fellow to E.D.I., summer fellowship to C.B.L., and Schaap Faculty Scholar to S.T.), NSF CAREER 0955975, ASMS Research Award (financially supported by Waters Corporation), Dupont Young Professor Award and Eli Lilly Young Investigator Award in Analytical Chemistry (to S.T.), as well as the Richard Houghton endowment to the University of the Sciences and NSF CHE-1112289 (to C.N.M.)

\section{References}

1. Niu, S., Zhang, W., Chait, B.T.: Direct Comparison of Infrared and Ultraviolet Wavelength Matrix-Assisted Laser Desorption/Ionization Mass Spectrometry of Proteins. J. Am. Soc. Mass Spectrom. 9, 1-7 (1997)

2. Jaskolla, T.W., Karas, M.: Compelling Evidence for Lucky Survivor and Gas Phase protonation: The Unified MALDI Analyte Protonation Mechanism. J. Am. Soc. Mass Spectrom. 22, 976-988 (2011)

3. Alves, S., Fournier, F., Afonso, C., Wind, F., Tabet, J.-C.: Gas-phase Ionization/desolvation Processes and their Effect on Protein Charge State Distribution under Matrix-assisted Laser Desorption/ionization Conditions. Eur. J. Mass Spectrom. 12, 369-383 (2006)

4. Chang, W.C., Huang, L.C.L., Wang, Y.-S., Peng, W.-P., Chang, H.C., Hsu, N.Y., Yang, W.B., Chen, C.H.: Matrix-Assisted Laser Desorption/ Ionization (MALDI) Mechanism Revisited. Analytica Chim. Acta 582, 1-9 (2007)

5. Knochenmuss, R.: Ion Formation Mechanisms in UV-MALDI. Analyst 131, 966-986 (2006)

6. Knochenmuss, R., Zhigilei, L.V.: Molecular Dynamics Model of Ultraviolet Matrix-assisted Laser Desorption/Ionization Including Ionization Processes. J. Phys. Chem. B 109, 22947-22957 (2005)

7. Knochenmuss, R., Zenobi, R.: MALDI Ionization: The Role of Inplume Processes. Chem. Rev. 103, 441-452 (2003)

8. Karas, M., Kruger, R.: Ion Formation in MALDI: The Cluster Ionization Mechanism. Chem. Rev. 103, 427-439 (2003)

9. Fournier, I., Brunot, A., Tabet, J.-C., Bolbach, G.: Delayed Extraction Experiments using a Repulsive Potential before Ion Extraction: Evidense of Clusters as Ion Precursors in UV-MALDI. Part 1: Dynamical Effects with the Matrix 2,5-dihydroxybenzoic Acid. Int. J. Mass Spectrom. 213, 203-215 (2002)

10. Karas, M., Gluckmann, M., Schafer, J.: Ionization in Matrix-assisted Laser Desorption/Ionization: Singly Charged Molecular Ions are the Lucky Survivors. J. Mass Spectrom. 35, 1-12 (2000)

11. Karas, M., Bachman, D., Bahr, U., Hillenkamp, F.: Matrix-Assisted Ultraviolet Laser Desorption of Non-Volatile Compounds. Int. J. Mass Spectrom. Ion Processes 78, 53-68 (1987)

12. Loboda, A.V., Krutchinsky, A.N., Bromirski, M., Ens, W., Standing, K.G.: A Tandem Quadrupole/Time-of-flight Mass Spectrometer witha Matrix Assisted Laser Desorption/Ionization Source Design and Performance. Rapid Commun. Mass Spectrom. 14, 1047-1057 (2000)

13. Baldwin, M.A., Medzihradszky, K.F., Lock, C.M., Fisher, B., Settinen, T., Burlingame, A.L.: Matrix-Assisted Laser Desorption/Ionization Coupled with Quadrupole/Orthogonal Acceleration Time-of-Flight Mass Spectrometry for Protein Discovery, Identification, and Structural Analysis. Anal. Chem. 73, 1707-1720 (2001)

14. Laiko, V.V., Moyer, S.C., Cotter, R.J.: Atmospheric Pressure MALDI/ Ion Trap Mass Spectrometry. Anal. Chem. 72, 5239-5243 (2000)

15. Laiko, V.V., Baldwin, M.A., Burlingame, A.L.: Atmospheric Pressure Matrix-Assisted Laser Desorption/Ionization Mass Spectrometry. Anal. Chem. 72, 652-657 (2000) 
16. Landgraf, R.R., Conaway, M.C.P., Garrett, T.J., Stacpoole, P.W., Yost, R.A.: Imaging of Lipids in Spinal Cord Using Intermediate Pressure Matrix-Assisted Laser Desorption-Linear Ion trap/Orbitrap MS. Anal. Chem. 81, 8488-8495 (2009)

17. Trimpin, S., Inutan, E.D., Herath, T.N., McEwen, C.N.: Laserspray Ionization - A New AP-MALDI Method for Producing Highly Charged Gas-Phase Ions of Peptides and Proteins Directly from Solid Solutions. Mol. Cell. Proteom. 9, 362-367 (2010)

18. Trimpin, S., Inutan, E.D., Herath, T.N., McEwen, C.N.: Matrix-Assisted Laser Desorption/Ionization Mass Spectrometry Method for Selectively Producing Either Singly or Multiply Charged Molecular Ions. Anal. Chem. 82, 11-15 (2010)

19. Lietz, C.B., Richards, A.L., Ren, Y., Trimpin, S.: Inlet Ionization: Protein Analyses from the Solid State Without the Use of a Voltage or a Laser Producing 67 Charges on BSA, 66 kDa. Rapid Commun. Mass Spectrom. 25, 3453-3456 (2011)

20. Schneider, B.B., Lock, C., Covey, T.R.: AP and Vacuum MALDI on a Qqlit Instrument. J. Am. Soc. Mass Spectrom. 16, 176-182 (2005)

21. Tanaka, K., Waki, H., Ido, Y., Akita, S., Yoshida, Y.: Protein and Polymer Analysis up to $m / z$ 100,000 by Laser Ionization Time-of-flight Mass Spectrometry. Rapid Commun. Mass Spectrom. 2, 151-153 (1988)

22. Karas, M., Hillenkamp, F.: Laser Desorption Ionization of Proteins with Molecular Masses Exceeding 10,000 Daltons. Anal. Chem. 60, 22992301 (1988)

23. McEwen, C.N., Pagnotti, V.S., Inutan, E.D., Trimpin, S.: New Paradigm in Ionization: Multiply Charged Ion Formation from a Solid Matrix without a Laser or Voltage. Anal. Chem. 82, 9164-9168 (2010)

24. Pagnotti, V.S., Chubatyi, N.D., McEwen, C.N.: Solvent Assisted Inlet Ionization: An Ultrasensitive New Liquid Introduction Ionization Method for Mass Spectrometry. Anal. Chem. 83, 3981-3985 (2011)

25. Pagnotti, V.S., Inutan, E.D., Marshall, D.D., McEwen, C.N., Trimpin, S.: Inlet Ionization: A New Highly Sensitive Approach for Liquid Chromatography-Mass Spectrometry of Small and Large Molecules. Anal. Chem. 82, 7591-7594 (2011)

26. Wang, B., Inutan, E.D., Trimpin, S.: A New Approach to High Sensitivity Liquid Chromatography-Mass Spectrometry of Peptides using Nanoflow Solvent Assisted Inlet Ionization MS/MS. J. Am. Soc. Mass Spectrom. 23, 442-445 (2012)

27. Ehring, H., Karas, M., Hillenkamp, F.: Role of photoionization and photochemistry in ionization processes of organic molecules and relevance for matrix-assisted laser desorption lonization mass spectrometry. Org. Mass Spectrom. 27, 472-480 (1992)

28. Dreisewerd, K., Schurenberg, M., Karas, M., Hillenkamp, F.: Influence of the Laser Intensity and Spot Size on the Desorption of Molecules and Ions in Matrix-assisted Laser Desorption/ionization with a Uniform Beam Profile. Int. J. Mass Spectrom. 141, 127-148 (1995)

29. Vestal, M.L.: Ionization Techniques for Nonvolatile Molecules. Mass Spectrom. Rev. 2, 447-480 (1983)

30. Dodd, E.E.: The Statistics of Liquid Spray and Dust Electrification by the Hopper and Laby Method. J. Appl. Phys. 24, 73-81 (1953)

31. Iribarne, J.V.: Thomson BA: On the Evaporation of Small Ions from Charged Droplets. J. Chem. Phys. 64, 2287-2294 (1976)

32. Hillenkamp, F.: Private Communication. 2011

33. Kinsel, G.R., Kinsel, M.E., Gillig, K.J., Russell, D.H.: Investigation of the dynamics of matrix-assisted laser desorption/ionization ion formation using an electrostatic analyzer/time-of-flight mass spectrometer. $J$. Mass Spectrom. 34, 684-690 (1999)

34. Zhigilei, L.V., Garrison, B.J.: Microscopic Mechanism of Laser Ablation of Organic Solids in the Thermal and Stress Confinement Irradiation Regimes. J. Appl. Phys. 88, 1281-1298 (2000)

35. Krutchinsky, A.N., Chait, B.T.: On the Nature of the Chemical Noise in MALDI Mass Spectra. J. Am. Soc. Mass Spectrom. 13, 129-134 (2002)

36. Zhigilei, L.V.: Dynamics of the Plume Formation and Parameters of the Ejected Clusters in Short-pulse Laser Ablation. Appl. Phys. A 76, 339350 (2003)

37. Handschuh, M., Nettesheim, S., Zenobi, R.: Laser-induced molecular desorption and particle ejection from organic films. Appl. Surf. Sci. 137, 125-135 (1999)

38. Cai, Y., Peng, W.-P., Kuo, S.J., Sabu, S., Han, C.C., Chang, H.C.: Optical Detection and Charge State Analysis of MALDI-Generated Particles with Molecular Masses Greater than 5 MDa. Anal. Chem. 74, 4434-4440 (2002)
39. McEwen, C.N., Trimpin, S.: An Alternative Paradigm in Mass Spectrometry: Flying Elephants from Trojan Horses. Int. J. Mass Spectrom. 300, 167-172 (2011)

40. Musapelo, T., Murray, K.K.: Particle Formation in Ambient MALDI Plumes. Anal. Chem. 83, 6601-6608 (2011)

41. Fournier, F., Brunot, A., Tabet, J.-C., Bolbach, G.: Delayed Extraction Experiments Using a Replusing Potential Before Ion Extraction: Evidence of Noncovalent Clusters as Ion Precursor in UV Matrixassisted Laser Desorption/ionization. Part II-Dynamic Effect with Alpha-cyano-4-hydroxycinnamic Acid Matrix. J. Mass Spectrom. 40, 50-59 (2005)

42. Inutan, E.D., Wang, B., Trimpin, S.: Commercial Intermediate Pressure MALDI Ion Mobility Spectrometry Mass Spectrometer Capable of Producing Highly Charged Laserspray Ionization Ions. Anal. Chem. 83, 678-684 (2011)

43. McEwen, C.N., Larsen, B.S., Trimpin, S.: Laserspray Ionization on a Commercial AP-MALDI Mass Spectrometer Ion Source: Selecting Singly or Multiply Charged Ions. Anal. Chem. 82, 4998-5001 (2010)

44. Trimpin, S., Jing, L., Wang, B., Lietz, C.B., Green, D.R., Manly, C.D., Richards, A.L., Marshall, D.D., Ren, Y., Lingenfelter, S., Inutan, E.D.: Matrix Assisted Ionization: New Aromatic and Non Aromatic Matrix Compounds Producing Multiply Charged Lipid, Peptide, and Protein Ions in the Positive and Negative Mode Obseved Directly from Surfaces. J. Am. Soc. Mass Spectrom. (2012). doi:10.1007/s13361021-0413-z

45. Trimpin, S., Ren, Y., Wang, B., Lietz, C.B., Richards, A.L., Marshall, A.G., Inutan, E.D.: Extending the Laserspray Ionization Concept to Produce Highly Charged Ions at High Vacuum on a Time-of-Flight Mass Analyzer. Anal. Chem. 83, 5469-5475 (2011)

46. Karas, M., Bahr, U., Stupat, K., Hillenkamp, F., Tsarhpoulos, A., Pramanik, B.N.: Matrix Dependence of Metastable Fragmentation of Glycoproteins in MALDI TOF Mass Spectrometry. Anal. Chem. 67, 675-679 (1995)

47. Wang, B., Lietz, C.B., Inutan, E.D., Leach, S., Trimpin, S.: Laserspray Ionization Ion Mobility Spectrometry Mass Spectrometry: A Total Solvent-free Analysis Approach at Atmospheric Pressure. Anal. Chem. 83, 4076-4084 (2011)

48. Dole, M., Mack, L.L., Hines, R.L., Mobley, R.C., Ferguson, L.D., Alice, M.B.: Molecular Beams of Macroions. J. Chem. Phys. 49, 22402249 (1968)

49. Inutan, E.D., Trimpin, S.: Laserspray Ionization (LSI) Ion Mobility Spectrometry (IMS) Mass Spectrometry (MS) J. Am. Soc. Mass Spectrom. 21, 1260-1264 (2010)

50. Inutan, E.D., Richards, A.L., Wager-Miller, J., Mackie, K., McEwen, C.N., Trimpin, S.: Laserspray Ionization-A New Method for Protein Analysis Directly from Tissue at Atmospheric Pressure and with UltraHigh Mass Resolution and Electron Transfer Dissociation Sequencing. Mol. Cell. Proteomics 110, 1-8 (2011)

51. Fenn, J.B.: Electrospray Wings for Molecular Elephants. Angewandte Chemie-Int. Ed. 42, 3871-3894 (2003)

52. Page, J.S., Tang, K., Kelly, R.T., Smith, R.D.: Subambient Pressure Ionization with Nanoelectrospray Source and Interface for Improved Sensitivity in Mass Spectrometry. Anal. Chem. 80, 1800-1805 (2008)

53. Richards, A.L., Lietz, C.B., Wager-Miller, J., Mackie, K., Trimpin, S.: Localization and imaging of gangliosides in mouse brain tissue sections by laserspray ionization inlet. J. Lipid Res. 53, 1390-1398 (2012)

54. Trimpin, S.: A Perspective on MALDI Alternatives - Total Solvent-free Analysis and Electron Dissociation of Highly Charged Ions by Laserspray Ionization. J. Mass Spectrom. 45, 471-485 (2010)

55. Richards, A.L., Lietz, C.B., Trimpin, S.: Imaging Mass Spectrometry in Transmission Geometry. Rapid Commun. Mass Spectrom. 25, 815-829 (2011)

56. Sardelis, D.L., Pagnotti, V.S., McEwen, C.N., Bentzley, C.M.: Investigating the Instrumental Optimization and Sensitivity of Laserspray Ionization Inlet for Protein Analysis. Proceedings 59th ASMS Conference on Mass Spectrometry and Allied Topics, Denver, June, MP01:352 (2011)

57. Latham, J., Stow, C.D.: The Mechanism of Charge Transfer Associated with Evaporation of Ice. J. Atmos. Sci. 23, 245-247 (1968)

58. Thomas, R.J., Krehbiel, P.R., Rison, W., Edens, H.E., Aulich, G.D., Winn, W.P., McNutt, S.R., Tytgat, G., Clark, E.: Electrical Activity During the 2006 Mount St. Augustine Volcanic Eruptions. Science 315, 1097 (2007) 
59. Luts, A., Parts, T.-E., Laakso, L., Hirsikko, A., Gronhohm, T., Kuhmala, M.: Some Air Electricity Phenomena Cause by Waterfalls: Correlative Study of the Spectra. Atmospheric Research 91, 229-237 (2009)

60. Suzuki, M., Asano, K.: A Mathematical Model of Droplet Charging in Ink-Jet Printers. J Phys D: Appl. Phys. 12, 529-537 (1979)

61. Chapman, S.: Carrier Mobility Spectra of Liquids Electrified by Bubbling. Phys. Rev. 54, 520-527 (1938)

62. Workman, E.J., Reynolds, S.E.: Electrical Phenomena Occurring During the Frezing of Dilute Aqueous Solution and Their Possible Relationship to Thunderstorm Electricity. Phys. Rev. 78, 254-259 (1950)

63. Saunders, C.: Charge Separation Mechanisms in Clouds. Space Sci. Rev. 137, 335-353 (2008)

64. Gibson, H.W.: Linear Free Energy Relationships. V. Triboelectric Charging of Organic Solids. J. Am. Chem. Soc. 97, 3832-3833 (1975)

65. Yates, D.E., Levine, S., Healy, T.W.: Site-binding Model of the Electrical Double Layer at the Oxide/Water Interface. J. Chem. Soc. faraday Trans. 1(70), 1807-1818 (1974)

66. Vaaraslahti, K., Laitinen, A., Keskinen, J.: Spray Charging of Droplets in a Wet Scrubber. J. Air Waste Manag. Assoc. 52, 175-180 (2002)

67. Sunner, J., Ikonomou, M.G., Kebarle, P.: SIMS Spectra of Alcohols and the Phase Explosion Model of Desorption Ionization. Int. J. Mass Spectrom. Ion Processes 82, 221-237 (1988)

68. Kosevich, M.V., Shelkovsky, V.S., Boryak, O.A., Orlov, V.V.: 'Bubble Chamber Model' of Fast Atom Bombardment Induced Processes. Rapid Commun. Mass Spectrom. 17, 1781-1792 (2003)

69. Mo, Q., Detwiler, A.G., Helsdon, J.H., Winn, W.P., Aulich, G.D., Murray, W.C.: Hydrometeor Charges Observed Below an Electrified Cloud Using a New Instrument. J. Geophys. Res. 112, D13207-D13220 (2007)

70. Zilch, L.W., Maze, J.T., Smith, J.W., Ewing, G.F., Jarrold, M.F.: Charge Separation in the Aerodynamic Breakup of Micrometer-Sized Water Droplets. J. Phys. Chem. A 112, 13352-13363 (2008)

71. Lenard, P.: Über Wasserfallelektrizität und über die Oberflächenbeschaffenheit der Flüssigkeiten. Ann. Physik. 352, 463-524 (1915)

72. Zubarev, R.A., Hakansson, P., Sundqvist, B., Talrose, V.L.: Enhancement of the Molecular Ion Yield in Plasma Desorption Mass Spectrometry Using Explosive Matrices. Rapid Commun. Mass Spectrom. 11, 63-70 (1997)

73. Hakansson, K., Zubarev, R.A., Coorey, R.V., Talrose, V.L., Hakansson, P.: Interaction Between Explosive and Analyte Layers in Explosive Matrix-assisted Plasma Desorption Mass Spectrometry. Rapid Commun. Mass Spectrom. 13, 1169-1174 (1999)

74. Reyesa, A., Domi'ngueza, R.M., Tostaa, M., Herizea, A., Chuchania, G.: Kinetics of the Gas-phase Homogeneous Unimolecular Elimination of Selected Ethyl Esters of 2-Oxo-carboxylic Acids. J. Phys. Org. Chem. 24, 74-82 (2011)

75. Schmidt, A.-C., Herzschuh, R., Matysik, F.-M., Engewald, W.: Investigation of the Ionisation and Fragmentation Behaviour of Different Nitroaromatic Compounds Occurring as Polar Metabolites of Explosives using Electrospray Ionisation tandem Mass Spectrometry. Rapid Commun. Mass Spectrom. 20, 2293-2302 (2006)

76. Li, J., Brill, T.B.: Spectroscopy of Hydrothermal Reactions 23: The Effect of OH Substitution on the Rates and Mechanisms of Decarboxylation of Benzoic Acid. J. Phys. Chem. A 107, 2667-2673 (2003)

77. Alves, S., Kalberer, M., Zenobi, R.: Direct Detection of Particles Formed from Laser Ablation of Matrices During Matrix-Assisted Laser
Desoprtion/Ionization. Rapid Commun. Mass Spectrom. 17, 2034-2038 (2003)

78. Wu, X., Sadeghi, M., Vertes, A.: Molecular Dynamics of MatrixAssisted Laser Desorption of Leucine Enkephalin Guest Molecules from nicotinic Acid Host Crystal. J. Phys. Chem. B 104, 4770-4778 (1998)

79. Kristyan, S., Bencsura, A., Vertes, A.: Modeling the Cluster Formation During Infrared and Ultraviolet Matrix-Assisted Laser Desorption Ionization of Oligonucleotides in Succinic Acid Matrix with Molecular Mechanics. Theoret. Chim. Acta 107, 319-325 (2002)

80. Chubatyi, N.D., McEwen, C.N.: Improving Ion Production and Efficiency with Obstruction Surfaces using Sonic Spray Ionization (SSI) Mass Spectrometry. Proceedings of the 59th ASMS Conference on Mass Spectrometry and Allied Topics, Denver, June, TP04:065 (2011)

81. Daub, C.D., Cann, N.M.: How Are Completely Desolvated Ions Produced in Electrospray Ionization: Insights from Molecular Dynamics Simulations. Anal. Chem. 83, 8372-8376 (2011)

82. Merenbloom, S.I., Flick, T.G., Williams, E.R.: How Hot are Your Ions in TWAVE Ion Mobility Spectrometry? J. Am. Soc. Mass Spectrom. 23, 553-562 (2012)

83. Allwood, D.A., Dreyfus, R.W., Perera, I.K., Dye, P.E.: UV Optical Absorption of Matrices Used for Matrix-assisted Laser Desorption/ Ionization. Rapid Commun. Mass Spectrom. 10, 1575-1578 (1996)

84. Bae, Y.J., Moon, J.H., Kim, M.S.: Expansion Cooling in the Matrix Plume is Under-Recognized in MALDI Mass Spectrometry. J. Am. Soc. Mass Spectrom. 22, 1070-1078 (2011)

85. Inutan, E.D., Wang, B., Wager-Miller, J., Mackie, K., Trimpin, S.: A Commercial Intermediate Pressure MALDI-IMS-MS Producing Multiply Charged Laserspray Ionization Ions. Proceedings of the 59th ASMS Conference on Mass Spectrom. and Allied Topics, Denver, June, MPO1:016 (2011)

86. Knochenmuss, R., Vertes, A.: Time-delayed 2-Pulse Studies of MALDI Matrix Ionization Mechanisms. J. Phys. Chem. B 104, 5406-5410 (2000)

87. Fenn, J.B.: Ion Formation from Charged Droplets: The Roles of Geometry, Energy, and Time J. Am. Soc. Mass Spectrom. 4, 524-535 (1993)

88. Ehring, H., Costa, C., Demirev, P.A., Sundqvist, B.U.R.: Photochemical Versus Thermal Mechanisms in Matrix-assisted Laser Desorption/ Ionization Probed by Back Side Desorption. Rapid Commun. Mass Spectrom. 10, 821-824 (1996)

89. Wortmann, A., Pimenova, T., Alves, S., Zenobi, R.: Investigation of the First Shot Phenomenon in MALDI Mass Spectrometry of Protein Complexes. Analyst 132, 199-207 (2007)

90. Moon, J.H., Shin, Y.-S., Bae, Y.J., Kim, M.S.: Ion Yields for Some Salts in MALDI: Mechanism for the Gas-Phase Ion Formation from Preformed Ions. J. Am. Soc. Mass Spectrom. 23, 162-170 (2012)

91. Mora, D.L., Van Berkel, G.J., Enke, C., Cole, R.B., Martinez-Shanchez, M., Fenn, J.B.: Electrospray Processes in Electrospray Ionization Mass Spectrometry. J. Mass Spectrom. 35, 939-952 (2000)

92. Trimpin, S.: Imaging Mass Spectrometry (MS) at Atmospheric and Intermediate Pressure using Laserspray Ionization (LSI) Proceedings of the 59th ASMS Conference on Mass Spectrometry and Allied Topics, Denver, June, MODam:10:10 (2011)

93. Crick, F.: What Mad Pursuit: A Personal View of Scientific Discovery. Alfred. P. Sloan. Foundation Series 59-60 (1988) 